\title{
Variación espacio-temporal de la comunidad de macroinvertebrados epibiontes en las raíces del mangle rojo Rhizophora mangle (Rhizophoraceae) en la laguna costera de La Mancha, Veracruz, México
}

\author{
Marcela Ruiz ${ }^{1} \&$ Jorge López-Portillo ${ }^{2}$
}

1. Centro de Investigación en Alimentación y Desarrollo, A.C., Unidad en Acuicultura y Manejo Ambiental (CIAD), Av. Sábalo-Cerritos s/n, CP 82100, Mazatlán, Sinaloa, México; marcela@ciad.mx

2. Red de Ecología Funcional, Instituto de Ecología, A.C. (INECOL), Carretera antigua a Coatepec 351, El Haya, CP 91070, Xalapa, Veracruz, México; jorge.lopez.portillo@inecol.mx

\author{
Recibido 02-XII-2013. Corregido 20-V-2014. Aceptado 24-VI-2014.
}

\begin{abstract}
Spatial-temporal variation of the macroinvertebrate epibiont community on red mangrove prop roots Rhizophora mangle (Rhizophoraceae) at La Mancha coastal lagoon, Veracruz, Mexico. La Mancha lagoon is connected to the Gulf of Mexico through an estuarine ephemeral inlet, a sand bar, which opens during the rainy season and closes during the late fall and the winter storm season. As the lagoon fills up with the permanent stream flow into its Southern part, the water level increases and the sand bar opens up, releasing huge volumes of water into the ocean and leaving the previously flooded intertidal zone exposed to drying. In this study, we described the spatial and temporal variations of structure and composition of the community living on the surface of red mangrove prop roots, related to the hydrodynamic of the estuarine inlet. Four red mangrove prop roots were collected every three months, over an annual period, at ten sampling stations. Water temperature, salinity, dissolved oxygen, water depth and transparency were also measured at each sampling event. To describe the identity, abundance, biomass, and specific richness of epibiont coverage on the prop roots, measurements were taken at $10 \mathrm{~cm}$ intervals (herein called "horizons"). A total of 59632 specimens and a total biomass of $172 \mathrm{~g} / \mathrm{cm}^{2}$ were registered, and a maximum of eleven horizons presented at least one species of epibionts. Two species of polychaetes, 12 of mollusks and 14 of crustaceans were identified, with Mytilopsis leucophaeata as the most abundant, Ficopomatus miamiensis as the most common and Crassostrea rhizophorae with the highest contribution in biomass. Sampling effort was assessed using species accumulation curves, resulting that the efficiency was, in general, greater than $90 \%$ of the richness predicted by asymptotic models. The hydrodynamics of the estuarine inlet had a great influence on the environmental conditions of the lagoon and on the spatial and temporal variation of the epibiont community: (a) when the inlet is open, there is a North-South gradient in environmental parameters (b) when the inlet is closed and the water level increases, the epibionts especially the pioneer species F. miamiensis, occupied the highest horizons of prop roots, and (c) the abundance, biomass and richness of epibionts increased towards the deeper and less saline Southern part of the lagoon, as well as on permanently submerged horizons, and during the seasonal closure of the inlet. Variations in depth are determined by the hydrodynamic behavior of the estuarine inlet, suggesting that these factors are major biodiversity drivers of the epibiont community at La Mancha. Rev. Biol. Trop. 62 (4): 1309-1330. Epub 2014 December 01.
\end{abstract}

Key words: ephemeral estuarine inlet, water depth, abundance, biomass, specific richness, mollusks, crustaceans, polychaetes, species accumulation curves.

El manglar ofrece, entre otros importantes beneficios, hábitat propicio para el arribo y permanencia de numerosas especies de origen marino y terrestre. Las raíces aéreas del mangle rojo Rhizophora mangle L., por su forma casi cilíndrica, representan un substrato firme, protegido de los procesos de sedimentación y con gran superficie de contacto disponible para su colonización por comunidades epibiontes de algas y de invertebrados (Ellison \& Farnsworth, 1992; Hogarth, 1999). Sin embargo, los organismos sésiles deben competir por el 
espacio en ese substrato para establecerse, alimentarse y desarrollar su ciclo de vida (Little \& Kitching, 1996).

El conocimiento sobre las comunidades de epibiontes en las raíces aéreas de mangle rojo proviene principalmente de estudios realizados en el Mar Caribe, en los cuales se analizaron uno o varios de los diferentes taxones que las integran, como por ejemplo esponjas (Díaz \& Rützler, 2009; Wulff, 2012), briozoos (Creary, 2003), tunicados, celenterados y algas (Guerra Castro, Cruz Motta, \& Eloy, 2011); moluscos, crustáceos y anélidos (García Padilla \& Palacio, 2008; Prüsmann \& Palacio, 2008; Romero Murillo \& Polanía, 2008; Cedeño, Jiménez Prieto, Pereda \& Allen, 2010; Quirós \& Arias, 2013; Acosta Balbas, Betancourt Tineo, \& Prieto Arcas, 2014). Por otro lado, en las costas del Pacífico mexicano, se han estudiado las comunidades de cirrípedos (Salgado Barragán \& Hendrickx, 2002a), cangrejos braquiuros (Salgado Barragán \& Hendrickx, 2002b) e isópodos (García Guerrero \& Hendrickx, 2003).

Aunque los procesos que estructuran a las comunidades de epibiontes en manglares son diversos y complejos (Ellison, Farnsworth, \& Twilley, 1996; Farnsworth \& Ellison, 1996; Rützler \& Feller, 1996, 1999; Hogarth, 1999), las condiciones ambientales, como la salinidad, temperatura, turbidez, pluviosidad, $\mathrm{pH}$, granulometría, materia orgánica y vecindad con otros ecosistemas, son factores fundamentales en su estructura y composición (Farnsworth \& Ellison, 1996; Márquez \& Jiménez, 2002). Al respecto, Márquez \& Jiménez (2002) estudiaron los moluscos en el Golfo de Santa Fe en Venezuela, un sistema costero totalmente abierto al mar que recibe principalmente descargas del río Santa Fe, la menor riqueza de especies se asoció a la zona de más alta variación de la salinidad cerca de la desembocadura de este río. Algo similar se registró en la Laguna de Bocaripo, en Venezuela, que también tiene comunicación permanente con el mar, pero donde el aporte de agua dulce proviene fundamentalmente de la precipitación pluvial (Cedeño et al., 2010). En la boca del sistema, la abundancia y riqueza de moluscos y crustáceos es mayor e imperan condiciones marinas de salinidad y de temperatura relativamente moderadas, mientras que en el interior de la laguna el ambiente es hipersalino, de alta temperatura y la riqueza es menor. Por último, la Ciénaga $\mathrm{La}$ Boquilla, Colombia, cuenta con una boca permanente y con salinidad estable a lo largo del año, por lo que no hay diferencias espaciales importantes en las comunidades de epibiontes (Quiceno Cuartas \& Palacio Baena, 2008).

Un caso muy diferente es la laguna costera de La Mancha, en donde las principales entradas de agua marina y de agua dulce están en los extremos norte y sur del sistema, respectivamente. Además, la boca del sistema, conformada por una barra arenosa, se cierra y se abre estacionalmente, lo que influye en los niveles de inundación de este cuerpo costero. Al cerrarse, el nivel de inundación de la laguna aumenta por el aporte de agua continental, incrementando con ello la profundidad y el espacio vertical que puede ser ocupado por organismos acuáticos. Cuando la boca se abre, y se descargan grandes volúmenes de agua hacia el mar, una proporción significativa del área inundada se expone a la desecación. Este ciclo se repite varias veces en el año. En el presente estudio se describe y analiza la variación espacio-temporal de la comunidad de macroinvertebrados epibiontes en las raíces del mangle rojo en función de la dinámica hidrológica del sistema.

\section{MATERIALES Y MÉTODOS}

Área de estudio: La laguna costera de La Mancha (Fig. 1), se localiza en la planicie costera central del estado de Veracruz, en el Golfo de México (19³4'-19³6' N y 96²2'$\left.96^{\circ} 24^{\prime} \mathrm{W}\right)$. Posee una boca estuarina efímera (Lankford, 1977) y el estrechamiento en su parte central la separa en zona norte y sur. Mide aproximadamente $3 \mathrm{~km}$ en su eje norte-sur, el espejo de agua es de 135 ha y tiene 355 ha de manglares (Hernández Trejo, Priego Santander, López-Portillo \& Insunza Vera, 2006). El clima es cálido subhúmedo con lluvias en verano, la temperatura media anual está entre $22^{\circ}$ y $26^{\circ} \mathrm{C}$ 


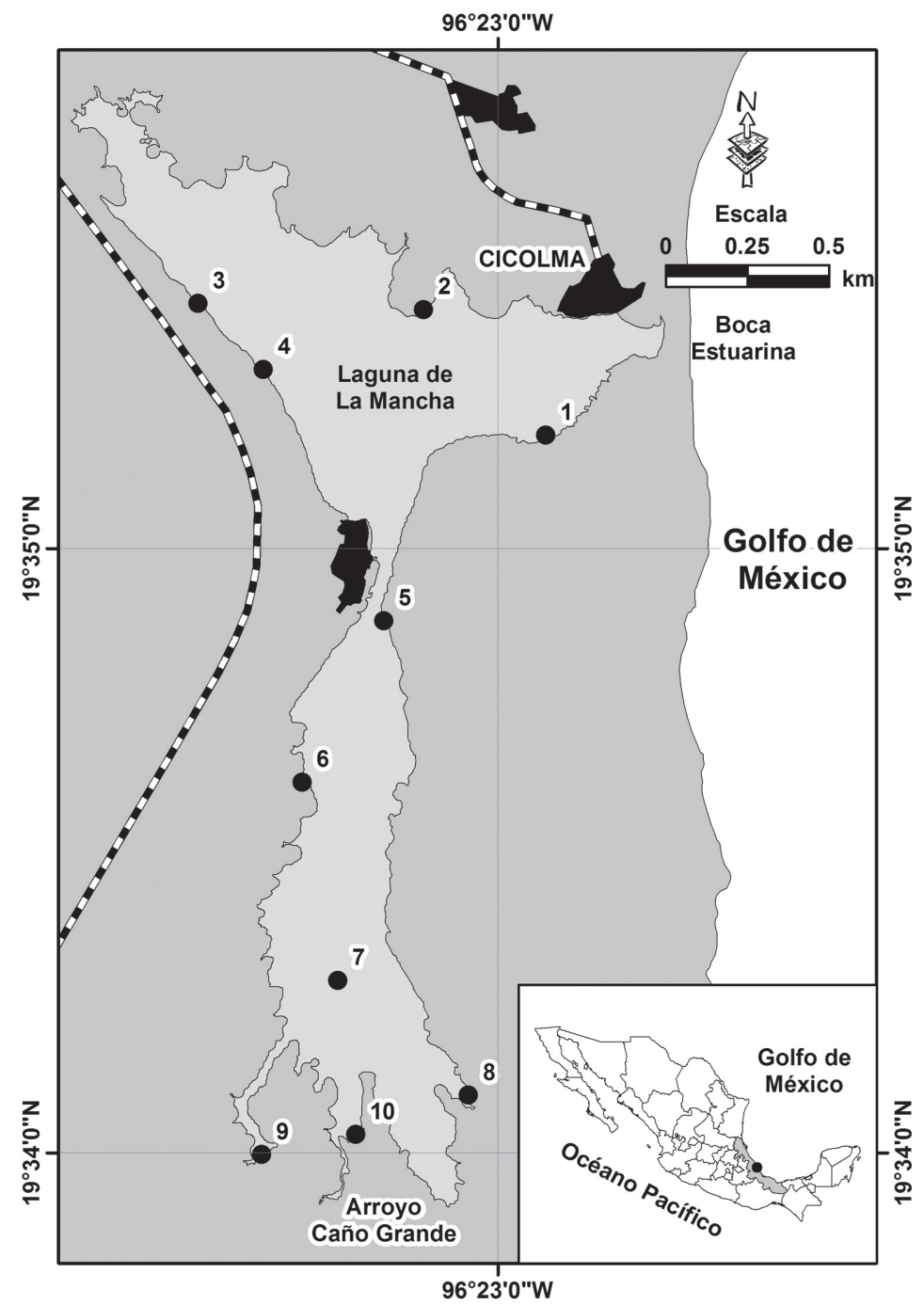

Fig. 1. Estaciones de muestreo en la Laguna La Mancha, Veracruz, México.

Fig. 1. Ten sampling stations located along the La Mancha lagoon study site, Veracruz, Mexico.

y la precipitación oscila entre 1200 y $1500 \mathrm{~mm}$ anuales (García, 1988). En esta región se distinguen tres períodos climáticos: lluvias (de junio a septiembre), nortes (frentes polares de finales de septiembre a marzo) y secas (que se traslapa con nortes, de finales de febrero a mayo). El aporte de agua marina a la laguna es a través de la boca en su parte noreste, mientras que el aporte principal de agua dulce es a través del arroyo Caño Grande, en el extremo sur. De manera cíclica, la boca estuarina se abre en época de lluvias y se cierra durante la época de nortes. Además de la apertura natural de la barra arenosa, en ocasiones los pescadores locales escarban un canal angosto en ella para abrirla; supuestamente, para evitar la muerte de ostiones y peces al disminuir la concentración de oxígeno en el interior (Lara Domínguez, Day, Yáñez Arancibia \& Sáinz Hernández, 2006). La laguna tiende a azolvarse (Flores Andolais, García Cubas \& Toledano Granados, 1988), lo que se refleja en su profundidad 
somera, de $0.20 \mathrm{~m}$ a $1 \mathrm{~m}$ en el norte, de 0.70 a $1.5 \mathrm{~m}$ en el centro, y hasta $2.5 \mathrm{~m}$ en el sur (Villalobos Figueroa et al., 1984).

Método de estudio: Se establecieron diez estaciones de muestreo en el interior de la laguna (Fig. 1), las estaciones uno a cuatro en la zona norte, con mangles rojos de 3 a $5 \mathrm{~m}$ de altura, y las estaciones cinco a diez en la zona sur, con árboles de $5 \mathrm{a} 7 \mathrm{~m}$ de altura. Las estaciones uno y dos son las más cercanas a la boca estuarina mientras que las estaciones nueve y diez son las más alejadas de ésta (Fig. 1).

Se llevaron a cabo cuatro muestreos (agosto y noviembre de 2000, febrero y mayo de 2001). En cada muestreo se registró si la boca estuarina estaba abierta o cerrada, y en cada estación de muestreo se midió in situ, por triplicado, la profundidad y transparencia del agua (con disco de Secchi graduado) y la salinidad, temperatura y oxígeno disuelto en el agua superficial, a $20 \mathrm{~cm}$ de profundidad, con sistemas portátiles de medición (instrumentos YSI-30 y YSI-55).

En cada estación de muestreo se marcaron 50 raíces en contacto con el sedimento del fondo y con forma y diámetro similares, de entre las cuales se seleccionaron al azar cuatro raíces en cada fecha de muestreo. Para extraerlas se hicieron dos cortes, uno al ras del fondo y otro a $20 \mathrm{~cm}$ por arriba del nivel de marea alta. Se hicieron observaciones in situ sobre la ubicación de las especies móviles en las raíces antes de cortar las raíces. Las raíces se colocaron individualmente en bolsas de polietileno y se llevaron al laboratorio del Centro de Investigaciones Costeras de La Mancha (CICOLMA), donde se mantuvieron en refrigeración a $4^{\circ} \mathrm{C}$ hasta su procesamiento.

En el laboratorio, cada raíz fue subdividida y analizada en segmentos de $10 \mathrm{~cm}$, llamándolos "horizontes", y numerándolos de la parte distal (en contacto con el sedimento) hacia la parte proximal al tallo y expuesta a la marea alta. Los especímenes se retiraron de la raíz horizonte por horizonte. Se procedió a separar las especies de poliquetos, moluscos y crustáceos del resto de los taxones, considerando como "macro invertebrado" a las especies mayores de $1 \mathrm{~mm}$ de longitud. Los especímenes de cada especie se cuantificaron y pesaron para cada horizonte de cada raíz. Una vez retirados todos los epibiontes, se midió el diámetro (d) y el largo (h) de cada raíz para calcular el área $(A)$ de la raíz aproximándola a un cilindro $(A=\pi \mathrm{dh})$.

Los moluscos se fijaron en formol al $6 \% \mathrm{y}$ después se conservaron en etanol al 70\%, y los crustáceos y poliquetos se depositaron directamente en etanol al 70\% (modificado de Gaviño, Juárez \& Figueroa, 1992). La determinación al nivel de especie fue realizada por especialistas basándose principalmente en la siguiente literatura: para poliquetos Rioja $(1945,1946)$, para moluscos Abbott (1974) y para crustáceos Felder (1973), García Cubas (1981), Schultz (1969) y Williams $(1983,1984)$. Los crustáceos y moluscos fueron depositados para su resguardo en el Laboratorio de Ecología Funcional en el INECOL, Xalapa, y los poliquetos en el Laboratorio de Poliquetos del ICMyL-UNAM, en la ciudad de México. El arreglo taxonómico se presenta según Brusca y Brusca (1990).

En cada raíz recolectada se determinó la abundancia ( $\mathrm{N}=$ número de individuos de cada especie), la biomasa $\left(\mathrm{Bm}=\mathrm{g} / \mathrm{cm}^{2}\right.$ por especie) y la riqueza específica ( $\mathrm{S}=$ número de especies) de los individuos presentes en cada muestreo, en cada estación y en cada horizonte. Adicionalmente, se determinó la frecuencia de aparición de las especies por raíces, así como la frecuencia temporal, espacial y vertical. En cada caso, la frecuencia fue calculada como el porcentaje del total de raíces, de muestreos, de estaciones y de horizontes en la que se registró cada especie. De esta manera, una especie con una frecuencia temporal de $25 \%$ significa que se recolectó en uno de los cuatro muestreos, o bien, una especie con una frecuencia espacial del $50 \%$, se colectó en cinco de las diez estaciones de muestreo. De acuerdo a la presencia de las especies en las diferentes estaciones, se determinó si pertenecen a la zona norte o sur de la laguna.

Eficiencia del muestreo: Se utilizó el método de funciones de acumulación de 
especies para estimar la riqueza de especies y la eficiencia del muestreo. Para ello se emplearon dos modelos asintóticos (Soberón \& Llorente, 1993). Los modelos suponen que la probabilidad de encontrar más especies disminuye (modelo de Dependencia lineal) o aumenta (modelo de Clench) conforme se incrementa el esfuerzo de muestreo. De hecho, el modelo de Dependencia lineal predice un límite inferior y el modelo de Clench el límite superior de los valores estimados, por lo que la curva de acumulación de especies observadas debe estar entre ambas predicciones (Moreno \& Halffter, 2000). Se evaluó la efíciencia del muestreo para cada una de las estaciones de muestreo, considerando como esfuerzo de muestreo a las raíces recolectadas a lo largo del año.

El orden en que fueron tomadas las muestras fue aleatorizado 100 veces con la rutina EstimateS (Colwell, 1997) para eliminar el efecto de la secuencia de las recolectas y obtener una curva suavizada. Los dos modelos se ajustaron a esta curva mediante una regresión no lineal. La eficiencia del muestreo se evaluó como el porcentaje que representan las especies observadas del total de las especies esperadas, calculado como la asíntota en ambos modelos.

Los datos de abundancia, biomasa y riqueza específica fueron sometidos a un análisis de varianza (ANOVA) de tres vías para probar si había diferencias entre muestreos, entre estaciones y entre horizontes, considerando cada raíz como réplica (Zar, 1996). Los datos fueron previamente transformados $\left(x^{\prime}=\log _{10}(x+1)\right)$, para cumplir con los supuestos de normalidad y homogeneidad de varianzas. En los casos donde existieron diferencias significativas entre factores se aplicó la prueba a posteriori de Duncan (Sokal \& Rohlf, 1979).

También se analizó el efecto del cierre y apertura de la boca estuarina sobre la cobertura vertical de los epibiontes en las raíces. Para ello, se llevó a cabo un ANOVA de tres vías para explorar las diferencias en la cobertura vertical de los diferentes taxones (poliquetos, moluscos y crustáceos), entre las estaciones de muestreo y entre las dos condiciones de la boca estuarina (abierta o cerrada), siendo la variable de respuesta el horizonte máximo ocupado por los epibiontes en cada raíz.

Por último, para explorar la asociación de la distribución espacial y temporal de las especies con los parámetros ambientales, se llevó a cabo un Análisis de Correspondencia Canónica (ACC), una técnica de análisis directo de gradientes (Ter Braak, 1986). Se prepararon dos matrices de datos, una conteniendo las cinco variables ambientales y otra conteniendo los valores de abundancia de todas las especies (el promedio de las cuatro raíces en todos sus horizontes para cada estación y fecha). Para este análisis se utilizó el programa MVSP, vers. 3.13c. Para cumplir con los supuestos de normalidad requeridos para el ACC, tanto los datos de abundancia como los datos ambientales se transformaron logarítmicamente $\left(x^{\prime}=\log 10(x+1)\right)$.

\section{RESULTADOS}

Caracterización ambiental: La boca estuarina se abrió cinco veces de octubre del 2000 a abril del 2001, cuatro por acción de los pescadores y una de manera natural. El tiempo en que la boca permaneció cerrada fluctuó de días a varias semanas. De los cuatro muestreos realizados solamente el de febrero coincidió con la boca estuarina cerrada.

En general, se registraron gradientes que van de valores más altos en el norte a valores más bajos en el sur, desde la boca estuarina hacia la desembocadura del Caño Grande, en todos los parámetros ambientales (Figs. 2 y 3). La laguna fue más profunda en febrero y somera en mayo (Fig. 2A). El valor medio mínimo de $13.3 \pm 1.5 \mathrm{~cm}$ (media \pm error estándar) se registró en la estación uno en mayo, y el medio máximo de $117.3 \pm 4.1 \mathrm{~cm}$ en la estación diez, en febrero. La columna de agua fue más transparente en febrero y menos transparente en agosto (Fig. 2B).

En agosto y febrero hubo poca variabilidad en la salinidad, mientras que en noviembre y especialmente en mayo se marcó el gradiente norte-sur (Fig. 3A). Resaltan valores hipersalinos en la estación uno en el mes de mayo 


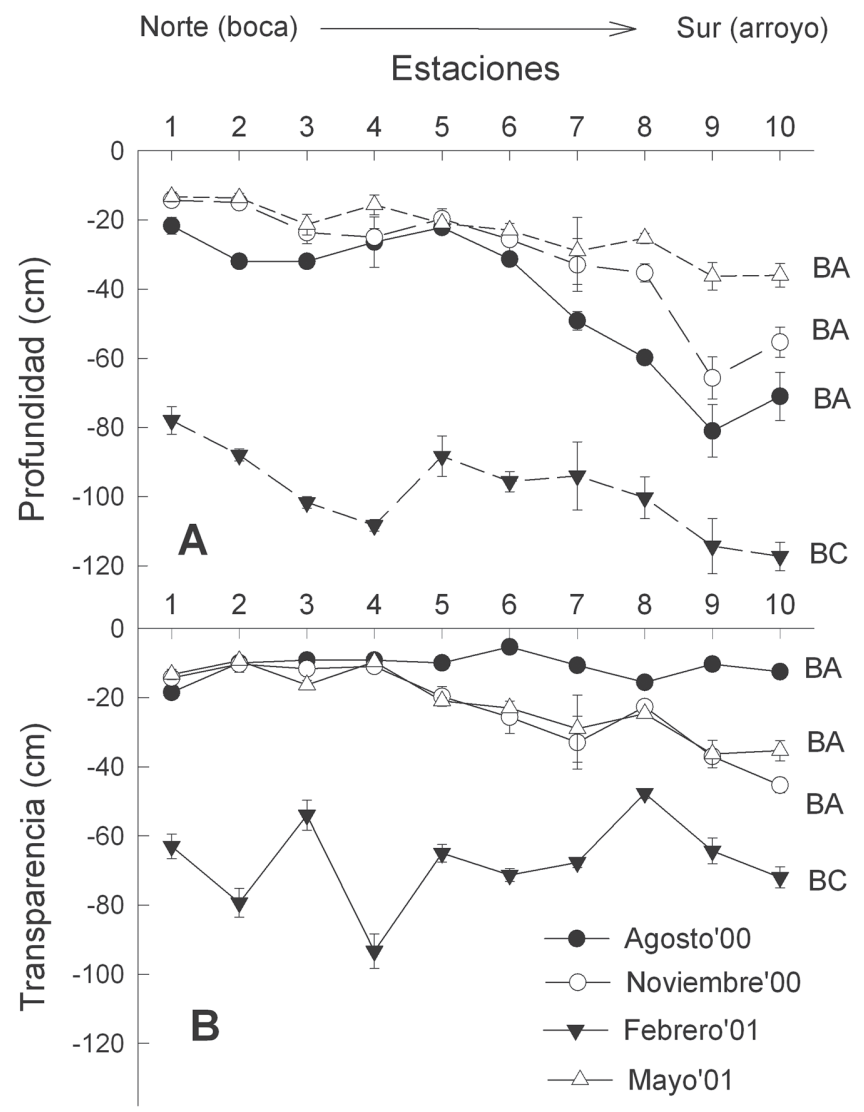

Fig. 2. Profundidad del agua (A) y transparencia (B) en cuatro muestreos y diez estaciones ubicadas desde la boca estuarina (norte) hasta la desembocadura del arroyo (sur), en la laguna de La Mancha. BA: Boca estuarina abierta; BC: Boca estuarina cerrada. Las barras verticales indican el error estándar.

Fig. 2. Water depth (A) and transparency (B) on four sampling dates at ten stations situated along the La Mancha lagoon from the estuarine inlet (North) to the stream outlet (South). BA: Open estuarine inlet; BC: Closed estuarine inlet. Vertical bars indicate one standard error.

$(58.6 \pm 0.49 \%$ ). La temperatura media mínima $\left(22.6 \pm 0.2^{\circ} \mathrm{C}\right)$ se registró en febrero en la estación diez y la media máxima $\left(34.9 \pm 0.03^{\circ} \mathrm{C}\right)$, en mayo en la estación uno (Fig. 3B). La concentración de oxígeno fue mayor en mayo en la estación uno $\left(10.1 \pm 0.6 \mathrm{mgO}_{2} / \mathrm{L}\right)$ y menor en noviembre en la estación diez $\left(2.4 \pm 0.5 \mathrm{mgO}_{2} / \mathrm{L}\right)$ (Fig. 3C).

Comunidad de epibiontes: Se procesaron 160 raíces con un máximo de once horizontes con ocupación de epibiontes. La comunidad estuvo compuesta por 28 especies de macroinvertebrados intermareales, de las cuales 14
(50\%) fueron Crustacea, 12 (43\%) Mollusca y dos (7\%) Polychaeta (Cuadro 1). Se cuantificaron 59632 individuos con una biomasa total de $172 \mathrm{~g} / \mathrm{cm}^{2}$. La especie más abundante fue Mytilopsis leucophaeata (Conrad 1831) (25 724 individuos) y la más rara Cerithidea pliculosa (Menke 1829) (un individuo). En términos de biomasa, la especie dominante fue Crassostrea rhizophorae (Guilding 1828) $\left(119 \mathrm{~g} / \mathrm{cm}^{2}\right)$ (Cuadro 1).

Crassostrea rhizophorae fue la especie con mayor frecuencia de aparición en las raíces (89\%) mientras que Ficopomatus miamiensis (Treadwell 1934) fue la única especie con 


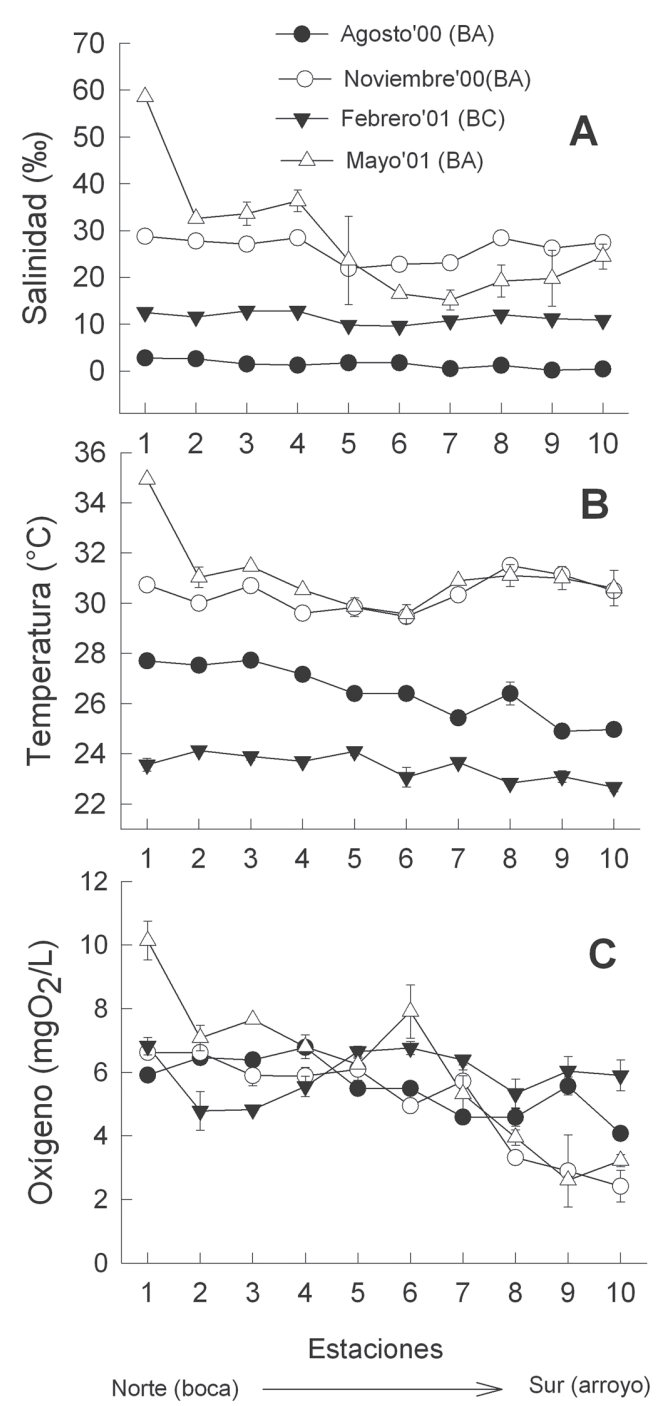

Fig. 3. Salinidad (A), temperatura (B), y oxígeno disuelto (C) en cuatro muestreos y diez estaciones, desde la boca estuarina (norte) hasta la desembocadura del arroyo (sur), en la laguna de La Mancha. BA: Boca estuarina abierta; $\mathrm{BC}$ : Boca estuarina cerrada. Las barras indican el error estándar.

Fig. 3. Salinity (A), temperature (B) and dissolved oxygen (C) on four sampling dates at ten stations situated along La Mancha lagoon from the estuarine inlet (North) to the stream outlet (South). BA: Open estuarine inlet; BC: Closed estuarine inlet. Vertical bars indicate one standard error.
$100 \%$ de frecuencia temporal, espacial y vertical (Cuadro 1). Un poco más de la mitad de las especies (16) tuvieron una frecuencia temporal de $100 \%$ y cuatro de ellas de $25 \%$. Estas últimas se recolectaron sólo en febrero.

Ocho especies registraron una frecuencia espacial de $100 \%$ y dos especies sólo se registraron en una estación (Cuadro 1). La mayoría de las especies sésiles estuvieron presentes en los primeros seis horizontes y varias de las especies móviles, como Littoraria angulifera (Lamarck, 1822) y Aratus pisonii (H. MilneEdwards, 1837), ocuparon también los horizontes siete y ocho cercanos al tallo. Diecinueve especies $(68 \%$ del total) estuvieron presentes en ambas zonas de la laguna, tres especies fueron exclusivas de la zona norte y seis especies de la zona sur (Cuadro 1).

Eficiencia del muestreo: El ajuste de los datos observados a los modelos matemáticos (Fig. 4) fue significativo para todas las estaciones de muestreo $\left(r^{2}>0.90, \mathrm{p}<0.001\right.$; Cuadro 2). La riqueza total registrada para las diez estaciones de muestreo fluctuó entre 14 a 21 especies, mientras que la predicción de los modelos arrojó un mínimo de 13 especies y un máximo de 23 especies por estación (Cuadro 2). Con el modelo de Dependencia lineal, el inventario de especies fue exitoso para las diez estaciones de muestreo, con una riqueza mayor al 100\% del límite inferior estimado por el modelo. Con el modelo de Clench, sólo en las estaciones uno, dos y cuatro los valores observados estuvieron por debajo del $90 \%$ de la predicción (Cuadro 2).

Variación temporal: La mayor abundancia y biomasa total de epibiontes se obtuvo en febrero (21 155 individuos, $64.55 \mathrm{~g} / \mathrm{cm}^{2}$ ) y la menor en noviembre ( 8625 individuos, $20.63 \mathrm{~g} /$ $\mathrm{cm}^{2}$ ) (Fig. 5). La riqueza específica fue menor en agosto (19 especies) y mayor en febrero y mayo (24 especies, Fig. 5C). 


\section{CUADRO 1}

Abundancia total, biomasa total, frecuencia de aparición (por raíces, temporal, espacial y vertical) y presencia por zonas de los macroinvertebrados epibiontes de las raíces de mangle rojo en La Mancha, México

TABLE 1

Total abundance, total biomass, frequency of occurrence (by roots, temporal, spatial and vertical) and presence by zones of macroinvertebrates epibionts of prop roots of red mangrove at La Mancha, Mexico

\begin{tabular}{|c|c|c|c|c|c|c|c|}
\hline Especie & $\begin{array}{l}\text { Abundancia } \\
\text { Total } \\
\text { (núm. ind.) }\end{array}$ & $\begin{array}{l}\text { Biomasa } \\
\text { Total } \\
\left(\mathrm{g} / \mathrm{cm}^{2}\right)\end{array}$ & $\begin{array}{l}\text { Frecuencia } \\
\text { por raíces } \\
(\% \text { de } n=160)\end{array}$ & $\begin{array}{l}\text { Frecuencia } \\
\text { temporal } \\
(\% \text { de } n=4)\end{array}$ & $\begin{array}{l}\text { Frecuencia } \\
\text { Espacial } \\
(\% \text { de } n=10)\end{array}$ & $\begin{array}{l}\text { Frecuencia } \\
\text { vertical } \\
(\% \text { de } n=11)\end{array}$ & $\begin{array}{l}\text { Presencia } \\
\text { por zonas } \\
\text { de la laguna }\end{array}$ \\
\hline $\begin{array}{l}\text { PHYLUM ANNELIDA } \\
\text { Clase Polychaeta } \\
\text { (poliquetos, gusanos marinos, gusanos tubícolas) } \\
\text { Familia Nereididae }\end{array}$ & & & & & & & \\
\hline $\begin{array}{l}\text { Nereis pelagica occidentalis Hartman, } 1945 \\
\text { Familia Serpulidae }\end{array}$ & 182 & 0.0539 & 27.5 & 100 & 100 & 55 & Norte, sur \\
\hline $\begin{array}{l}\text { Ficopomatus miamiensis (Treadwell, 1934) } \\
\text { PHYLUM MOLLUSCA } \\
\text { Clase Gastropoda (caracoles) } \\
\text { Familia Neritidae }\end{array}$ & 6137 & 0.4163 & 38.7 & 100 & 100 & 100 & Norte, sur \\
\hline $\begin{array}{l}\text { Puperita tristis (Orbigny, 1842) } \\
\text { Neritina reclivata (Say, 1822) } \\
\text { Neritina virginea (Linnaeus, 1758) }\end{array}$ & $\begin{array}{c}130 \\
197 \\
3415\end{array}$ & $\begin{array}{l}0.0734 \\
0.1088 \\
1.5395\end{array}$ & $\begin{array}{c}22.5 \\
32.5 \\
55\end{array}$ & $\begin{array}{l}100 \\
100 \\
100\end{array}$ & $\begin{array}{l}60 \\
80 \\
90\end{array}$ & $\begin{array}{l}45 \\
55 \\
64\end{array}$ & $\begin{array}{l}\text { Sur } \\
\text { Norte, sur } \\
\text { Norte, sur }\end{array}$ \\
\hline $\begin{array}{l}\text { Familia Littorinidae } \\
\text { Littoraria angulifera (Lamarck, 1822) } \\
\text { Familia Hidrobiidae }\end{array}$ & 16 & 0.0843 & 8.7 & 100 & 60 & 73 & Norte, sur \\
\hline $\begin{array}{l}\text { Littoridina sphinctostoma Abbott y Ladd, } 1951 \\
\text { Familia Potamididae }\end{array}$ & 5 & 0.00008 & 2.5 & 50 & 30 & 36 & Sur \\
\hline $\begin{array}{l}\text { Cerithidea pliculosa (Menke, 1829) } \\
\text { Familia Pyramidellidae }\end{array}$ & 1 & 0.000025 & 0.6 & 25 & 10 & 9 & Norte \\
\hline $\begin{array}{l}\text { Boonea impressa (Say, 1822) } \\
\text { Clase Bivalvia (ostiones, mejillones, horadadores) } \\
\text { Familia Mytilidae }\end{array}$ & 16 & 0.00057 & 4.4 & 75 & 60 & 27 & Norte, sur \\
\hline $\begin{array}{l}\text { Ischadium recurvum (Rafinesque, 1820) } \\
\text { Familia Pteriidae }\end{array}$ & 5265 & 31.3117 & 70.6 & 100 & 100 & 55 & Norte, sur \\
\hline $\begin{array}{l}\text { Isognomon alatus (Gmelin, 1791) } \\
\text { Familia Ostreidae }\end{array}$ & 30 & 0.1465 & 5 & 75 & 40 & 27 & Norte, sur \\
\hline $\begin{array}{l}\text { Crassostrea rhizophorae (Guilding, 1828) } \\
\text { Familia Dreissenidae }\end{array}$ & 8748 & 119.0643 & 89.4 & 100 & 100 & 55 & Norte, sur \\
\hline $\begin{array}{l}\text { Mytilopsis leucophaeata (Conrad, 1831) } \\
\text { Familia Teredinidae }\end{array}$ & 25724 & 4.1782 & 61.9 & 100 & 100 & 73 & Norte, sur \\
\hline $\begin{array}{l}\text { Bankia fimbriatula Moll y Roch, } 1931 \\
\text { PHYLUM ARTHROPODA } \\
\text { SUBPHYLUM CRUSTACEA } \\
\text { Clase Maxillopoda } \\
\text { Subclase Cirripedia (balanos) } \\
\text { Familia Balanidae }\end{array}$ & 14 & 0.0423 & 5 & 75 & 50 & 27 & Norte, sur \\
\hline $\begin{array}{l}\text { Amphibalanus eburneus (Gould, 1841) } \\
\text { Amphibalanus amphitrite (Darwin, 1854) } \\
\text { Semibalanus cf. balanoides (Linnaeus, 1767) }\end{array}$ & $\begin{array}{c}8595 \\
2 \\
12\end{array}$ & $\begin{array}{c}13.7838 \\
0.0011 \\
0.0009\end{array}$ & $\begin{array}{l}80.6 \\
1.2 \\
3.1\end{array}$ & $\begin{array}{l}100 \\
25 \\
75\end{array}$ & $\begin{array}{l}100 \\
20 \\
30\end{array}$ & $\begin{array}{l}64 \\
18 \\
27\end{array}$ & $\begin{array}{l}\text { Norte, sur } \\
\text { Norte } \\
\text { Norte }\end{array}$ \\
\hline $\begin{array}{l}\text { Clase Malacostraca } \\
\text { Superorden Eucarida } \\
\text { Orden Decapoda } \\
\text { Infraorden Caridea (camarones pistola) } \\
\text { Familia Palaemonidae }\end{array}$ & & & & & & & \\
\hline $\begin{array}{l}\text { Palaemonetes vulgaris (Say, 1818) } \\
\text { Infraorden Anomura (cangrejos ermitaños) } \\
\text { Familia Diogenidae }\end{array}$ & 12 & 0.0018 & 5 & 100 & 50 & 27 & Norte, sur \\
\hline Clibanarius vittatus (Bosc, 1802) & 3 & 0.0111 & 1.2 & 50 & 20 & 18 & Sur \\
\hline
\end{tabular}


CUADRO 1 (Continuación) / TABLE 1 (Continued)

\begin{tabular}{|c|c|c|c|c|c|c|c|}
\hline Especie & $\begin{array}{l}\text { Abundancia } \\
\text { Total } \\
\text { (núm. ind.) }\end{array}$ & $\begin{array}{c}\text { Biomasa } \\
\text { Total } \\
\left(\mathrm{g} / \mathrm{cm}^{2}\right)\end{array}$ & $\begin{array}{c}\text { Frecuencia } \\
\text { por raíces } \\
(\% \text { de } n=160)\end{array}$ & $\begin{array}{c}\text { Frecuencia } \\
\text { temporal } \\
(\% \text { de } n=4)\end{array}$ & $\begin{array}{c}\text { Frecuencia } \\
\text { Espacial } \\
(\% \text { de } n=10)\end{array}$ & $\begin{array}{l}\text { Frecuencia } \\
\text { vertical } \\
(\% \text { de } n=11)\end{array}$ & $\begin{array}{l}\text { Presencia } \\
\text { por zonas } \\
\text { de la laguna }\end{array}$ \\
\hline \multicolumn{8}{|l|}{ Infraorden Brachyura (cangrejos verdaderos) } \\
\hline \multicolumn{8}{|l|}{ Familia Panopeidae } \\
\hline Rhithropanopeus harrisii (Gould, 1841) & 85 & 0.1715 & 11.9 & 100 & 40 & 36 & Sur \\
\hline Eurypanopeus depressus (Smith, 1869) & 26 & 0.0091 & 6.9 & 75 & 60 & 27 & Norte, sur \\
\hline Panopeus lacustris Desbonne, 1867 & 30 & 0.1049 & 12.5 & 75 & 60 & 36 & Norte, sur \\
\hline \multicolumn{8}{|l|}{ Familia Grapsidae } \\
\hline Pachygrapsus gracilis (Saussure, 1858) & 560 & 0.6467 & 76.2 & 100 & 100 & 64 & Norte, sur \\
\hline \multicolumn{8}{|l|}{ Familia Sesarmidae } \\
\hline Aratus pisonii (H. Milne-Edwards, 1837) & 33 & 0.3278 & 16.9 & 100 & 80 & 73 & Norte, sur \\
\hline \multicolumn{8}{|l|}{ Superorden Peracarida } \\
\hline \multicolumn{8}{|l|}{ Orden Isopoda (isópodos, cochinillas de mar) } \\
\hline \multicolumn{8}{|l|}{ Familia Sphaeromatidae } \\
\hline Cassidinidea ovalis (Say, 1818) & 225 & 0.0018 & 38.1 & 100 & 100 & 55 & Norte, sur \\
\hline \multicolumn{8}{|l|}{ Orden Amphipoda (anfipodos) } \\
\hline \multicolumn{8}{|l|}{ Familia Gammaridae } \\
\hline Gammarus mucronatus Say, 1818 & 18 & 0.00031 & 1.2 & 25 & 10 & 18 & Sur \\
\hline \multicolumn{8}{|l|}{ Familia Aoridae } \\
\hline Grandidierella bonnieroides Stephensen, 1947 & 3 & 0.000053 & 1.9 & 25 & 20 & 9 & Sur \\
\hline \multicolumn{8}{|l|}{ Familia Melitidae } \\
\hline Melita nitida Smith, 1873 & 148 & 0.007 & 17.5 & 100 & 90 & 45 & Norte, sur \\
\hline Total: & 59632 & 172.0877 & & & & & \\
\hline
\end{tabular}

\section{CUADRO 2}

Número de especies observadas y parámetros de los modelos de Dependencia Lineal y de Clench utilizados para estimar la riqueza de epibiontes en diez estaciones de muestreo en la Laguna de La Mancha

TABLE 2

Number of recorded species and parameters of the Linear Dependence and Clench's models to predict the richness of the epibionts in ten sampling stations at La Mancha lagoon

\begin{tabular}{|c|c|c|c|c|c|c|c|c|c|c|c|}
\hline \multirow{2}{*}{ Estación } & \multirow{2}{*}{$\begin{array}{c}\text { Especies } \\
\text { observadas }\end{array}$} & \multicolumn{5}{|c|}{ Modelo de Dependencia Lineal } & \multicolumn{5}{|c|}{ Modelo de Clench } \\
\hline & & a & $\mathrm{b}$ & $\mathrm{a} / \mathrm{b}$ & $\%$ & $r^{2}$ & a & $\mathrm{b}$ & $\mathrm{a} / \mathrm{b}$ & $\%$ & $r^{2}$ \\
\hline 1 & 16 & 3.32 & 0.21 & 15.88 & 101 & 0.99 & 4.09 & 0.20 & 20.67 & 77 & 0.99 \\
\hline 2 & 19 & 4.61 & 0.25 & 18.22 & 104 & 0.98 & 6.10 & 0.27 & 22.57 & 84 & 0.99 \\
\hline 3 & 20 & 6.09 & 0.32 & 18.90 & 106 & 0.98 & 8.67 & 0.39 & 22.51 & 90 & 0.99 \\
\hline 4 & 17 & 5.80 & 0.35 & 16.57 & 103 & 0.99 & 8.27 & 0.42 & 19.59 & 87 & 0.99 \\
\hline 5 & 15 & 4.71 & 0.33 & 14.10 & 106 & 0.98 & 6.76 & 0.40 & 16.74 & 90 & 0.99 \\
\hline 6 & 17 & 5.56 & 0.36 & 15.56 & 109 & 0.95 & 8.33 & 0.46 & 18.19 & 93 & 0.98 \\
\hline 7 & 21 & 8.25 & 0.41 & 19.91 & 105 & 0.92 & 12.58 & 0.54 & 22.94 & 92 & 0.98 \\
\hline 8 & 21 & 7.56 & 0.38 & 19.64 & 107 & 0.90 & 11.48 & 0.50 & 22.80 & 92 & 0.97 \\
\hline 9 & 14 & 6.79 & 0.51 & 13.22 & 106 & 0.98 & 10.71 & 0.72 & 14.93 & 93 & 0.99 \\
\hline 10 & 16 & 10.45 & 0.69 & 15.21 & 105 & 0.97 & 17.79 & 1.06 & 16.72 & 95 & 0.99 \\
\hline & & & & romedio: & 105 & & & & omedio: & 89 & \\
\hline
\end{tabular}

a, b, son los parámetros de los modelos; a/b, es la asíntota; $\mathrm{r}^{2}$, es el coeficiente de determinación; \%, es el porcentaje que las especies recolectadas representan del total de las estimadas.

$\mathrm{a}, \mathrm{b}$, are the parameters of the models; $\mathrm{a} / \mathrm{b}$, asymptote; $\mathrm{r}^{2}$, coefficient of determination; $\%$, percent of collected species from the total predicted species. 


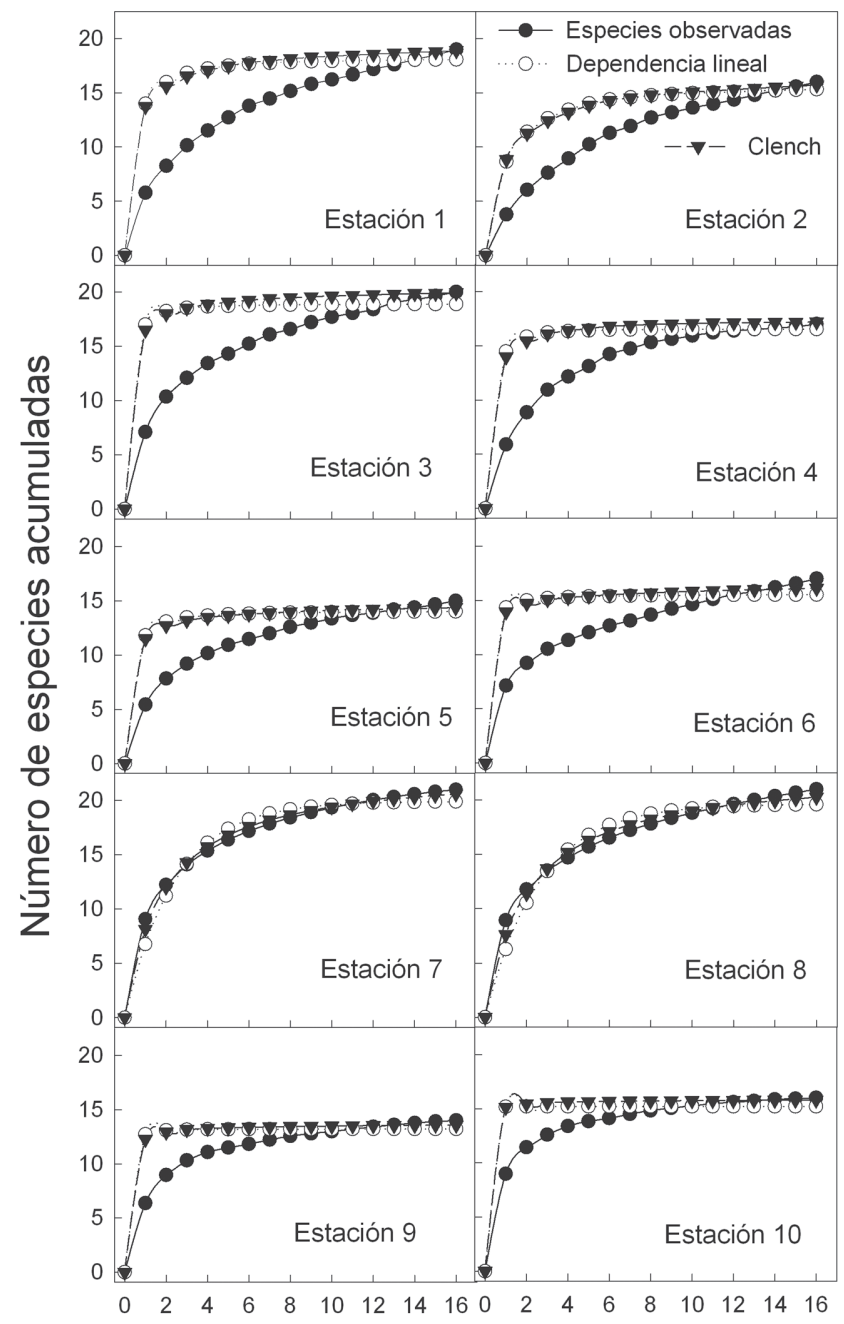

Esfuerzo de muestreo (Número de raíces)

Fig. 4. Curvas de acumulación de especies de las especies observadas y las predicciones de los modelos de Dependencia Lineal y de Clench de las diez estaciones de muestreo en la Laguna La Mancha.

Fig. 4. Species accumulation curves of species richness and Linear Dependence model and Clench model predictions of the ten sampling stations at La Mancha lagoon.

La abundancia y biomasa de moluscos fue mayor que la de crustáceos y poliquetos en los cuatro muestreos (Fig. 5), con un incremento notable en febrero (14 194 individuos, 61g/ $\left.\mathrm{cm}^{2}\right)$. La mayor riqueza correspondió a los crustáceos en febrero (12 especies). Las dos especies de poliquetos estuvieron presentes en los cuatro muestreos pero $F$. miamiensis proliferó significativamente en febrero (6 149 individuos, Fig. 5A). Hubo diferencias significativas entre muestreos y la prueba de Duncan indicó que la abundancia es similar en noviembre y mayo, la biomasa es similar en febrero y mayo, y la riqueza específica es diferente en los cuatro muestreos (Fig. 5).

Variación espacial: La abundancia total de epibiontes fue mayor en la estación diez, la 


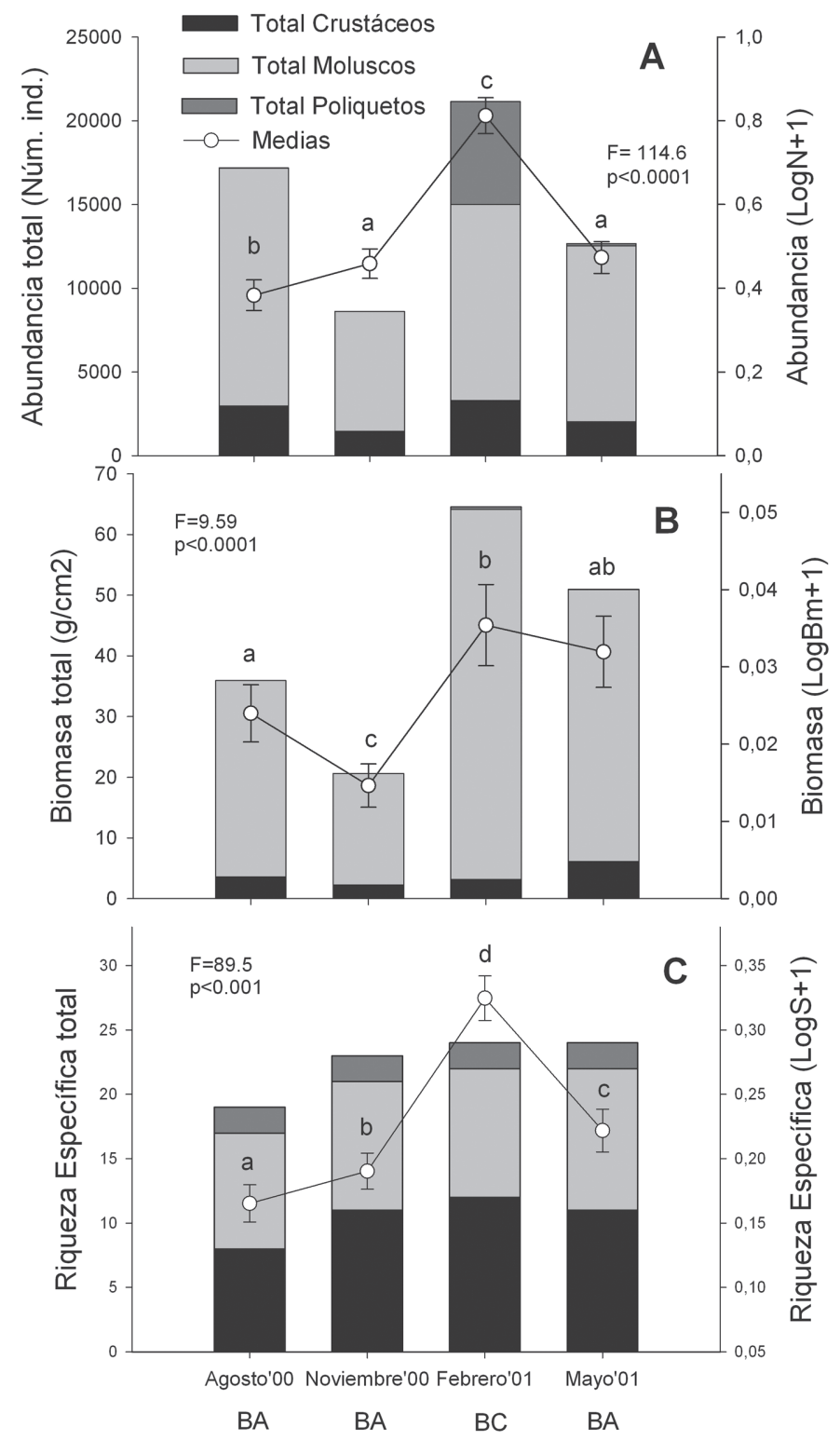

Fig. 5. Variación temporal de la abundancia (A), biomasa (B) y riqueza específica (C) de los epibiontes de las raíces del mangle rojo en la laguna de La Mancha. Las columnas indican valores totales y los valores de la media están representados por las líneas con marcadores. Las letras distintas corresponden a grupos homogéneos diferentes. Se indican los niveles de significancia para cada variable y las barras de los errores estándar. BA: Boca estuarina abierta; BC: Boca estuarina cerrada. Fig. 5. Temporal variation of abundance (A), biomass (B) and specific richness (C) of red mangrove prop-roots epibionts at La Mancha lagoon. The columns indicate total values and the media values are represented by lines and scatter plots. Different letters indicate different homogeneous groups. The significance levels for each variable and standard error bars are shown. BA: Open estuarine inlet; BC: Closed estuarine inlet. 
mayor biomasa total en la estación siete y la mayor riqueza específica total fue mayor en las estaciones tres, siete y ocho (Fig. 6). Aunque los moluscos predominaron en abundancia y biomasa, principalmente en las estaciones siete y diez, los crustáceos predominaron en riqueza específica en la mayoría de las estaciones (Fig. 6). Los poliquetos fueron abundantes en la estación diez (1 833 individuos).

De acuerdo con el ANOVA hubo diferencias significativas entre estaciones en las tres variables (Fig. 6). Los valores medios reflejan un gradiente que aumenta de norte a sur de la laguna, sobre todo en abundancia y riqueza. La prueba de Duncan formó diversos grupos homogéneos, especialmente en abundancia (siete grupos) y en riqueza específica (seis grupos). En general, las estaciones del norte son diferentes de las del sur excepto en biomasa, donde hay algunas similitudes entre ellas pero distinguiéndose la estación siete (Fig. 6).

Variación vertical: Se registró un máximo de once horizontes ocupados por epibiontes (110 cm por arriba del sedimento), pero el horizonte uno sobresale en biomasa total $(68.27 \mathrm{~g} /$ $\mathrm{cm}^{2}$ ) y el horizonte dos en abundancia y riqueza (19 266 individuos, 28 especies; Fig. 7). Los moluscos predominaron en abundancia y biomasa en los dos primeros horizontes mientras que los crustáceos predominaron en riqueza en el horizonte dos (14 especies). Los moluscos ocuparon hasta el horizonte nueve, los crustáceos hasta el ocho y el poliqueto $F$. miamiensis hasta el once. La prueba de Duncan posterior al ANOVA indicó que los horizontes más cercanos al fondo (uno al cinco) son diferentes entre sí en biomasa, abundancia y riqueza, mientras que los horizontes más altos (del seis al once) no difieren entre sí pero sí difieren del resto (Fig. 7).

Variación de la cobertura de epibiontes en función del cierre y apertura de la boca estuarina: Para analizar la cobertura vertical de los epibiontes sobre las raíces del mangle rojo en función de la hidrodinámica de la boca estuarina, se comparó entre los muestreos extremos de febrero (boca cerrada, laguna profunda) y de mayo (boca abierta, laguna somera) (Fig. 8). En febrero, la cobertura de los epibiontes se incrementó a horizontes altos, los moluscos ocuparon hasta el horizonte dos en las estaciones del norte, y hasta el horizonte siete en las estaciones del sur. Los crustáceos ocuparon del horizonte dos en la estación uno hasta el horizonte seis en la estación diez, y los poliquetos, del horizonte tres en la estación uno hasta el horizonte once en la estación diez (Fig. 8A).

En mayo, la cobertura de los epibiontes se concentró en los horizontes más cercanos al fondo, los moluscos y los crustáceos cubrieron el primer horizonte en la estación uno y un máximo de seis horizontes en la estación nueve. Los poliquetos estuvieron ausentes en la estación uno, y ocuparon hasta el horizonte uno en la estación dos y hasta el horizonte cuatro en la estación nueve (Fig. 8B).

El ANOVA indicó diferencias significativas en la cobertura vertical de epibiontes entre la boca estuarina abierta y cerrada $(\mathrm{F}=133.5$, $\mathrm{p}<0.0001)$, entre taxones $(\mathrm{F}=3.2, \mathrm{p}=0.042)$ $\mathrm{y}$ entre estaciones de muestreo $(\mathrm{F}=32.9$, $\mathrm{p}<0.0001$ ).

Relación entre la abundancia de epibiontes y los parámetros ambientales: Los autovalores del ACC fueron de 0.19 para el eje uno y de 0.11 para el eje 2, además estos dos ejes, en conjunto, explican alrededor del $30 \%$ de la variabilidad de los datos. La correlación de las especies y las variables ambientales con los ejes uno y dos fue relativamente alta $(0.88 \mathrm{y}$ 0.88 , respectivamente). La representación gráfica del eje 1 versus el eje 2 (Fig. 9), reveló la variación espacio-temporal de los parámetros ambientales y de las especies. En el eje 1, se expresó la variabilidad espacial, determinada por los gradientes ambientales y por una zonificación norte y sur de la laguna. Este eje estuvo positivamente correlacionado con la salinidad, oxígeno disuelto y la temperatura, y negativamente correlacionado con la profundidad y la transparencia. Por lo tanto, las estaciones del norte, más someras, mejor oxigenadas, más 

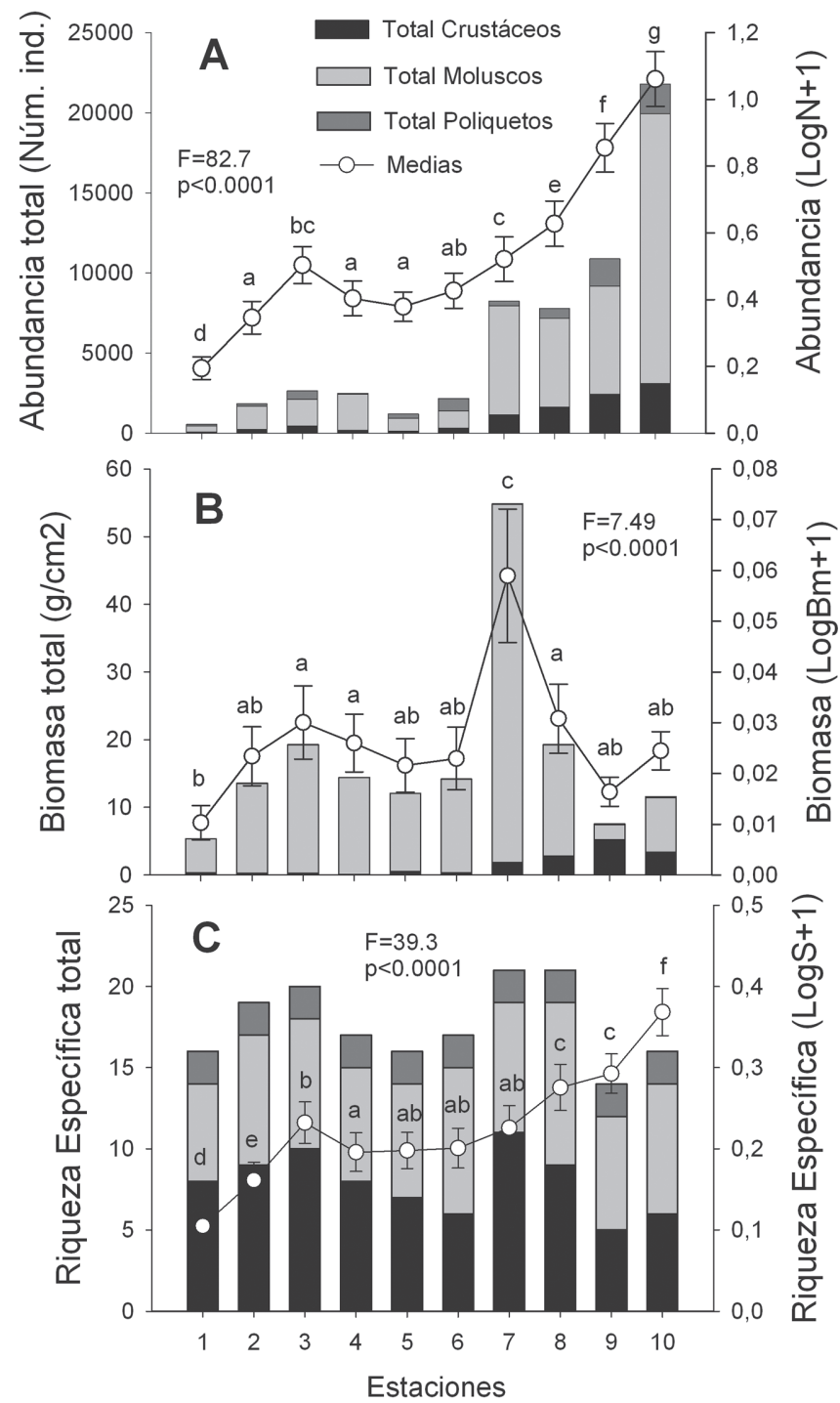

Norte (boca)

Sur (arroyo)

Fig. 6. Variación espacial de la abundancia (A), biomasa (B) y riqueza específica (C) de los epibiontes de las raíces de mangle rojo en la laguna de La Mancha. Las columnas indican valores totales y los valores de las medias están representados por las líneas con marcadores. Las letras distintas corresponden a grupos homogéneos diferentes. Se muestran los niveles de significancia para cada variable así como las barras de los errores estándar. Se especifica la dirección de la laguna, desde la boca estuarina (norte) hasta la desembocadura del arroyo (sur).

Fig. 6. Spatial variation of the abundance (A), biomass (B) and specific richness (C) of the red mangrove prop-roots epibionts at La Mancha lagoon. The columns indicate total values and the media values are represented by the lines and scatter plots. Different letters indicate different homogeneous groups. The significance levels for each variable and standard error bars are shown. The direction of the lagoon, from the estuarine inlet (North) to the stream outlet (South), is specified. 


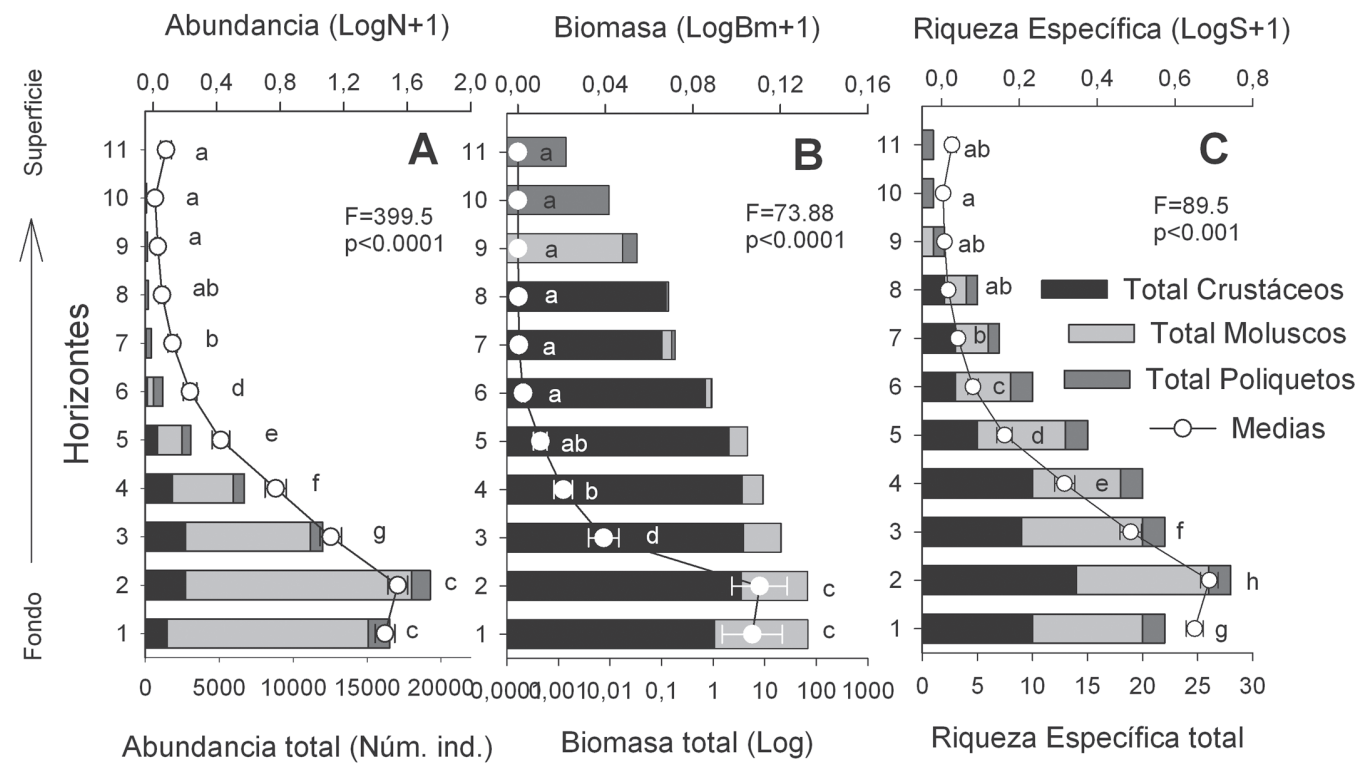

Fig. 7. Variación vertical de la abundancia (A), biomasa (B) y riqueza específica (C) de los epibiontes de las raíces del mangle rojo en la laguna de La Mancha. Las columnas indican valores totales y las líneas con marcadores, los valores de las medias. Las letras distintas corresponden a grupos homogéneos diferentes. Se muestran los niveles de significancia para cada variable así como las barras de los errores estándar. Se indica el gradiente vertical sobre las raíces desde el fondo lodoso hasta la superficie del agua.

Fig. 7. Vertical variation of the abundance (A), biomass (B) and specific richness (C) of the red mangrove prop-roots epibionts at La Mancha lagoon. The columns indicate total values and the lines and scatter plots the media values. Different letters indicate different homogeneous groups. The significance levels for each variable and standard error bars are shown. The vertical gradient on the prop roots from the muddy bottom to the water surface is indicated.

salinas y de mayor temperatura, se encuentran del lado derecho del eje uno, y las estaciones del sur, más profundas, menos oxigenadas, menos salinas y de menor temperatura están a la izquierda del mismo eje. Las especies relacionadas con la zona norte fueron especies de afinidad marina como Amphibalanus amphitrite (Darwin 1854) y Semibalanus balanoides Linnaeus 1767, mientras que las especies asociadas a la zona sur fueron $M$. leucophaeta, Neritina reclivata (Say, 1822), además de las que le son exclusivas por ser de afinidad dulceacuícola como Littoridina sphinctostoma Abbott y Ladd, 1951 y Rhithropanopeus harrisii (Gould, 1841).

El eje dos refleja la variabilidad temporal, con febrero en la parte superior (único muestreo con boca estuarina cerrada y valores altos de profundidad y transparencia) y los otros meses en la parte inferior (Fig. 9). Este eje estuvo positivamente correlacionado con la profundidad, la transparencia y el oxígeno, y negativamente correlacionado con la salinidad y la temperatura. En febrero destacó F. miamiensis y tres especies de anfípodos.

\section{DISCUSIÓN}

La variación en la profundidad siguió un patrón relacionado con los cambios estacionales de la boca estuarina: (a) durante la época de lluvias (agosto), el agua continental y el bajo aporte litoral de sedimentos mantuvieron abierta la boca estuarina, sólo incrementando la profundidad en breves lapsos; (b) durante la época de nortes, la boca estuarina se cerró y abrió ocasionalmente, oscilando la profundidad desde niveles relativamente bajos (noviembre), 


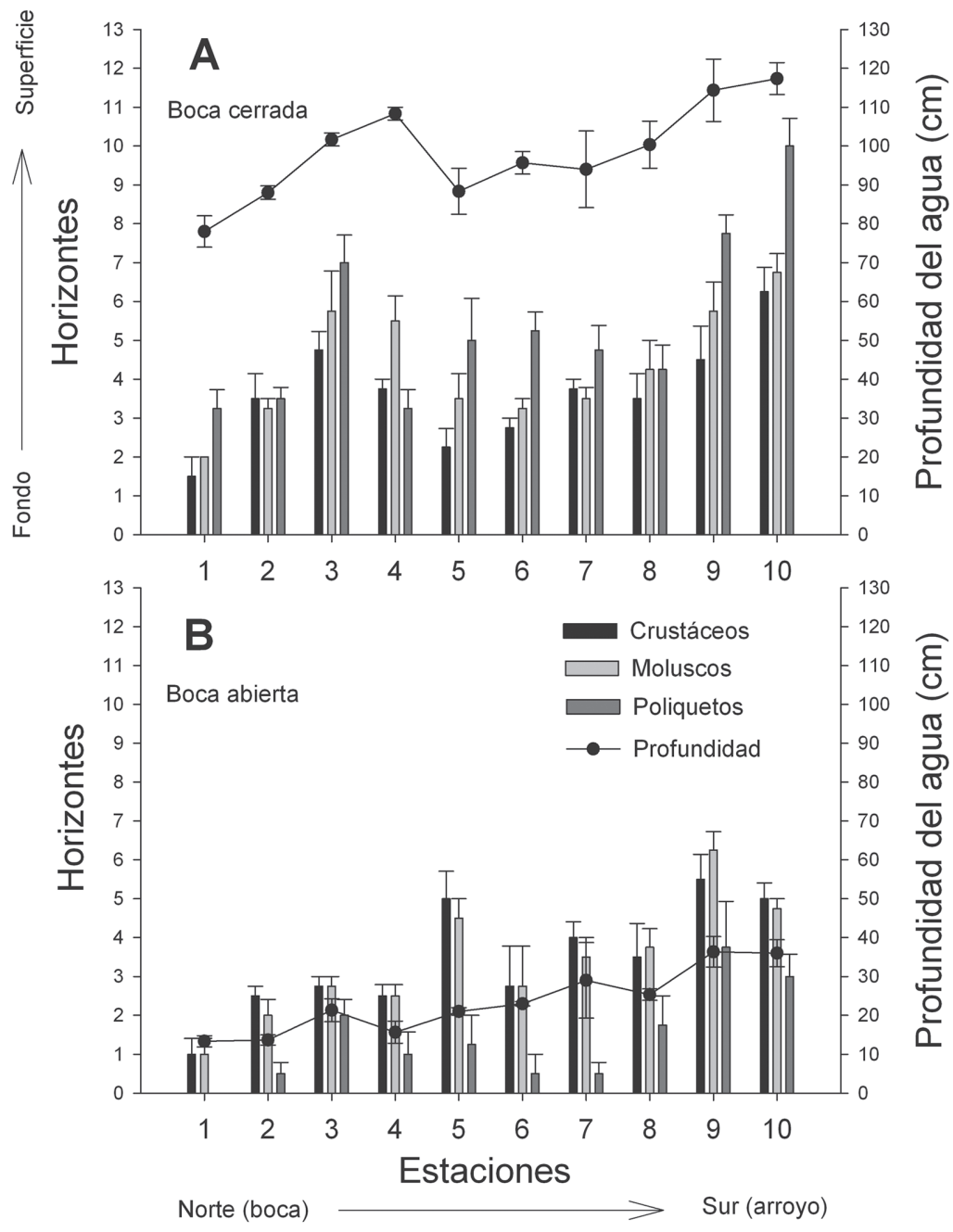

Fig. 8. Variación de la cobertura de crustáceos, moluscos y poliquetos sobre las raíces de mangle rojo y de la profundidad del agua, comparando muestreos de boca estuarina cerrada (febrero, A) y boca estuarina abierta (mayo, B), en diez estaciones de muestreo en la laguna de La Mancha. Se indica la dirección norte-sur de la laguna y el gradiente vertical sobre las raíces desde fondo lodoso a la superficie del agua. Se indican las barras de error estándar.

Fig. 8. Variation of the coverage of crustaceans, mollusks and polychaetes on the red mangrove prop-roots and of the water depth, comparing the period when the inlet is closed (February, A) and when it is open (May, B), at ten sampling stations at La Mancha lagoon. The North-South direction along the lagoon and the vertical gradient on the prop rooots from the muddy bottom to water surface, are indicated. The standard error bars are indicated.

hasta niveles máximos cuando ésta permaneció varias semanas cerrada (febrero); (c) durante la época de secas (mayo), se registraron las menores profundidades ya que el agua de escorrentía disminuyó y la boca estuarina estaba abierta. Las estaciones de muestreo más someras están en la zona más azolvada en el norte de la laguna (Villalobos Figueroa et al., 1984), mientras que las estaciones profundas coinciden con las áreas de descarga del arroyo Caño Grande.

La transparencia siguió el patrón estacional en el cual la columna de agua es menos transparente en época de lluvias debida a las descargas de arroyos y ríos, y es más transparente en secas, cuando no hay aportes importantes por escorrentía y el efecto de los vientos 


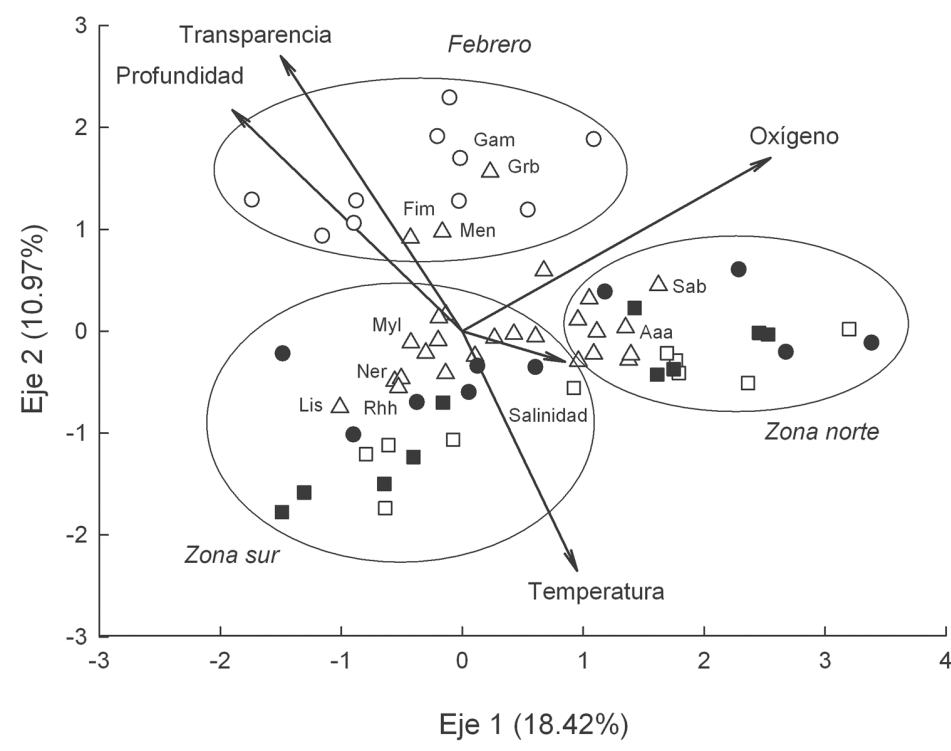

Fig. 9. Análisis de Correspondencia Canónica de la abundancia de epibiontes de las raíces del mangle rojo en cuatro fechas y diez estaciones de muestreo en la laguna de La Mancha. Triángulos blancos, especies; Círculos blancos, febrero; Círculos negros, mayo; Cuadrados blancos, agosto; Cuadrados negros, noviembre. Aaa, Amphibalanus amphitrite; Fim, Ficopomatus miamensis; Gab, Grandidierella bonnieroides; Gam, Gammarus mucronatus; Lis, Littoridina sphinctostoma; Men, Melita nitida; Myl, Mytilopsis leucophaeata; Ner, Neritina reclivata; Rhh, Rhithropanopeus harrisii; Sab, Semibalanus balanoides. Fig. 9. Canonical Correspondence Analysis of the abundance of red mangrove prop-roots epibionts on four sampling dates and ten sampling stations at La Mancha lagoon. White Triangles, species; White circles, February; Black circles, May; White squares, August; Black squares, November. Aaa, Amphibalanus amphitrite; Fim, Ficopomatus miamensis; Gab, Grandidierella bonnieroides; Gam, Gammarus mucronatus; Isa, Isognomon alatus; Lis, Littoridina sphinctostoma; Men, Melita nitida; Myl, Mytilopsis leucophaeata; Ner, Neritina reclivata; Rhh, Rhithropanopeus harrisii; Sab, Semibalanus balanoides.

es mínimo (Yáñez Arancibia \& Day, 2005). Igualmente, la variabilidad en la salinidad estuvo asociada a la estacionalidad de los aportes de agua marina y continental (Villalobos Figueroa et al., 1984; Yáñez Arancibia \& Day, 2005), a la dinámica hidrológica de la boca estuarina y al patrón de salinidad propuesto por Contreras Espinosa (1993) para sistemas estuarinos: las propiedades oligohalinas imperan en las desembocaduras de los ríos, las mesohalinas en las zonas de mezcla y las euhalinas en la zona de influencia marina.

La temperatura del agua en La Mancha es relativamente alta por ser una laguna tropical, pero hay una diferencia de más de $10^{\circ} \mathrm{C}$ entre el mes más frío (febrero) y el mes más cálido (mayo). Las altas temperaturas en mayo se explican por la mayor insolación aunada a una columna de agua somera, mucho más marcada en la zona norte (Villalobos Figueroa et al., 1984).

La concentración de oxígeno fue mayor en la parte norte de la laguna, lo que indica una alta tasa de recambio generada por la acción de los vientos sobre la columna de agua somera, y la circulación del agua por las mareas. La menor concentración de oxígeno al sur de la laguna puede deberse a un mayor caudal y aporte de sedimentos y partículas en suspensión (de la Lanza Espino, 1994). El gradiente de oxígeno descendente norte-sur en $\mathrm{La}$ Mancha también fue observado por Villalobos Figueroa et al. (1984).

En La Mancha, la comunidad de epibiontes está compuesta por especies típicas de ambientes estuarinos (García Cubas, 1981; 
Flores Andolais et al., 1988; Cruz Soto \& Jiménez, 1994). Es similar a las comunidades de las bahías del Turbo, El Uno (García Padilla \& Palacio, 2008) y de Cispatá (Quirós \& Arias, 2013), pero distinta de la Ciénaga la Boquilla donde la comunidad es más de origen marino (Quiceno Cuartas \& Palacio Baena, 2008). Sólo tres especies de moluscos, C. rhizophorae, Isognomon alatus Gmelin, 1791 y L. angulife$r a$, se comparten con el Golfo de Santa Fe, con la Ciénaga la Boquilla, la laguna de Bocaripo y la Bahía Mochima (Márquez \& Jiménez, 2002; Quiceno Cuartas \& Palacio Baena, 2008; Cedeño et al., 2010; Acosta Balbas et al., 2014). Además de C. rhizophorae y L. angulifera, también Neritina virginea (Linnaeus 1758) y Bankia fimbriatula Moll \& Roch, 1931 se comparten con la epifauna registrada en el Golfo de Urabá (García Padilla \& Palacio, 2008). En cuanto a los crustáceos, A. pisonii, Pachygrapsus gracilis Saussure, 1858, Amphibalanus eburneus Gould, 1841 y A. amphitrite son comunes con la laguna de Bocaripo (Cedeño et al., 2010) y el Golfo de Urabá (García Padilla \& Palacio, 2008). Los tres primeros también están presentes en la Bahía Cispatá (Quirós \& Arias, 2013). Eurypanopeus depressus (Smith 1869) es común con la Ciénaga la Boquilla (Quiceno Cuartas \& Palacio Baena, 2008) y con la Bahía de Cispatá (Quirós \& Arias, 2013). Por otro lado, ninguna de las dos especies de poliquetos registradas en La Mancha fueron citadas para las islas San Andrés, Colombia (Londoño Mesa, Polanía, \& Vélez, 2002; Romero Murillo \& Polanía, 2008), para el Golfo de Urabá (García Padilla \& Palacio, 2008), ni para la laguna La Restinga, en Venezuela (Díaz Díaz, Liñero Arana, Villafranca \& Allen, 2009).

La riqueza de epibiontes en el manglar de La Mancha (28 especies) es menor que la de los manglares del Caribe, algunos con más de 100 especies de invertebrados sésiles y algas (Ellison \& Farnsworth, 1992; Farnsworth \& Ellison, 1996; Rützler \& Feller, 1996; Rützler et al., 2000; Díaz \& Rützler, 2009; Cedeño et al., 2010). Sin embargo, para tal comparación se debe considerar las condiciones ambientales propias de cada sistema, los diferentes esfuerzos de muestreo, y especialmente la historia biogeográfica de cada región. Por ejemplo, las comunidades de raíces de mangle del Caribe, dominadas por esponjas y tunicados, son las más ricas en especies; las del este del Pacífico tienen abundantes balanos y baja cobertura de esponjas y tunicados, y las del Indo-Pacífico tienen mayor abundancia de ostiones (Hogarth, 1999). La composición y riqueza de especies de La Mancha son las esperadas para un sistema de manglar continental, con una menor riqueza de especies como respuesta a la alta turbidez y la variabilidad en la temperatura y salinidad del agua (Rützler \& Feller, 1996; Twilley, Snedaker, Yáñez Arancibia, \& Medina, 1996).

De acuerdo con Moreno y Halffter (2000), el $90 \%$ de la fauna estimada por cualquiera de los dos modelos asintóticos aplicados en este estudio, es un valor estandarizado aceptable para comparar la riqueza de especies entre comunidades. Con la aplicación del modelo más conservador, el modelo de Clench, podría deducirse que tres de las estaciones (uno, dos y cuatro), requerirían de un mayor esfuerzo de muestreo al presentar valores por debajo del 90\% de las especies estimadas. Sin embargo, hay que considerar que, particularmente la estación uno, es un sitio perturbado ubicado en el área azolvada de la boca estuarina lo que influye en una menor riqueza.

La raíz fue eficiente como unidad de muestreo, porque se aseguró la captura de los epibiontes sésiles al estar adheridos al substrato, y las especies móviles, que aunque migran verticalmente sobre la misma raíz siguiendo las oscilaciones de la marea (Wilson, 1981; Conde \& Díaz, 1989), se refugiaron en las conchas vacías de Crassostrea, por lo que muy pocos individuos escaparon o se cayeron durante la recolecta. Esta misma unidad de muestreo ha sido empleada en otros trabajos (Farnsworth \& Ellison, 1996; Salgado Barragán \& Hendrickx, 2002a, b; Márquez \& Jiménez, 2002; Vilardy \& Polanía, 2002; Quiceno Cuartas \& Palacio Baena, 2008; Cedeño et al., 2010. De estos autores, sólo Farnsworth \& Ellison (1996) presentan curvas de acumulación de especies pero no las ajustan a ningún modelo matemático para 
evaluar la eficiencia de su inventario. Aunque los autores recolectaron $\geq 50$ raíces por sitio, su muestreo parece ser eficiente con pocas muestras, pues encontraron casi el $90 \%$ de las especies en las raíces tomadas en los primeros $10-15 \mathrm{~m}$ de sus transectos de $50 \mathrm{~m}$. Por lo tanto, se considera que para La Mancha, la recolecta de 16 raíces por estación de muestreo fue aceptable para conocer la riqueza de la comunidad de epibiontes.

Los moluscos aportaron los mayores valores de abundancia y de biomasa en La Mancha. Particularmente C. rhizophorae, la especie con mayor biomasa, es común y abundante en otros sistemas costeros del Caribe (Márquez \& Jiménez, 2002; Quiceno Cuartas \& Palacio Baena, 2008; Quirós \& Arias, 2013; Acosta Balbas et al., 2014). Sin embargo, M. leucophaeata, la especie más abundante, no ha sido registrada en ninguno de los anteriores trabajos, pues se distribuye en el Golfo de México hasta Massachusetts (Marelli \& Gray, 1983), aunque es invasora de algunas costas europeas (Laine, Mattila, \& Lehikoinen, 2006) y brasileñas (Souza, Rocha, \& Lima, 2005). Por otra parte, F. miamiensis, una especie pionera y de rápido crecimiento, colonizó rápidamente los horizontes más altos en las raíces al aumentar el nivel de inundación en febrero, pero tras la apertura de la boca estuarina los individuos establecidos murieron por desecación.

En La Mancha, la estructura de la comunidad de epibiontes de las raíces del mangle rojo está asociada con todas las variables ambientales aquí medidas, según reveló el ACC, pero es importante notar que dichas variables dependen de la profundidad, y ésta a su vez está regulada por la hidrodinámica de la boca estuarina. Las mayores abundancias, biomasas y riquezas en las estaciones más profundas del sur, en los horizontes más profundos y durante la temporada que se cierra la boca estuarina, sugieren que el recurso limitante en esta laguna es el espacio nuevo, es decir, el substrato en dónde establecerse, al igual que lo registrado por Sutherland (1980) en Venezuela. Aun cuando el área sobre las raíces es menor cuando la boca estuarina está abierta, los epibiontes coexisten gracias a su capacidad de dispersión durante la etapa larval, y porque los hábitos gregarios y epifaunales de las especies sésiles brindan un hábitat diverso para las especies asociadas y móviles.

El efecto de la profundidad en la estructura de la comunidad de epibiontes no se ha analizado en otros trabajos. Probablemente, porque corresponden a sistemas de comunicación permanente con el océano, con profundidades más estables y con mayor influencia de la salinidad (Márquez \& Jiménez, 2002; Quiceno Cuartas \& Palacio Baena, 2008; García Padilla \& Palacio 2008; Cedeño et al., 2010; Ortiz \& Blanco, 2012; Quirós \& Arias, 2013).

Con los resultados aquí obtenidos se evidencia la importancia del funcionamiento de las bocas estuarinas y de su manejo adecuado. La apertura de bocas estuarinas por pescadores es una actividad común en México, que aún no está regulada en las normas oficiales mexicanas. Sin embargo, el gobierno federal está desarrollando un programa que incluye, entre otros aspectos, desazolves de áreas de productividad pesquera, rehabilitación de canales de navegación y estudios de impacto ambiental (SAGARPA, 2013), lo que abre la posibilidad de mejoramiento de la hidrología de algunas lagunas costeras mexicanas.

En conclusión, el funcionamiento de la boca estuarina regula las variaciones en la profundidad, mismas que estructuran la comunidad de moluscos, crustáceos y poliquetos epibiontes de las raíces del mangle rojo en la laguna de La Mancha. Cuando la boca estuarina está abierta son más evidentes los gradientes ambientales norte-sur. Hay una zonificación de la laguna en norte y sur, no sólo ocasionada por su forma de reloj de arena sino también de acuerdo a los parámetros ambientales, a la composición de especies y a las variables biológicas de los epibiontes. La abundancia, biomasa, riqueza específica y cobertura vertical de los epibiontes es mayor en el sur de la laguna, porque hay mayor disponibilidad de espacio sobre las raíces. 


\section{AGRADECIMIENTOS}

Este trabajo es parte de la tesis de Maestría en Ciencias de M. Ruiz. Fue financiado por CONACYT (259335-N) y se escribió con el apoyo del proyecto CREC (EU IRSES \#247514). Se agradece la ayuda en campo de H. Barradas López y F. Aguilar Santiago, y el apoyo en el laboratorio de J. Ramírez Gordillo, J. Tolome, V. Vásquez y R. Hernández. La determinación taxonómica fue realizada por A. Granados (Polychaeta), M. Reguero Reza (Mollusca) y A. Corona (Amphipoda) del ICMyL-UNAM-Unidad México; C. Espinoza (Isopoda) y J. Salgado Barragán (Decapoda y Cirripedia) del ICMyL-UNAM-Unidad Mazatlán; y A. Raz-Guzmán (Brachyura) de UMSNH-Morelia. Se agradece a los revisores anónimos que ayudaron a mejorar considerablemente este trabajo.

\section{RESUMEN}

La laguna de La Mancha está conectada al Golfo de México a través de una boca estuarina efímera, una barra arenosa, la cual se abre durante la época de lluvias y se cierra durante el otoño y la temporada de vientos invernales. Cuando la laguna se llena con la entrada de agua proveniente de un arroyo permanente, ubicado en la parte sur, el nivel del agua se incrementa y la barra arenosa se abre, descargando grandes volúmenes de agua hacia el océano y dejando expuesta a la desecación la zona intermareal previamente inundada. En este estudio, se describen las variaciones espacio-temporales de la estructura y composición de la comunidad de epibiontes que vive en la superficie de las raíces del mangle rojo, y su relación con la dinámica hidrológica de la boca estuarina. Se recolectaron cuatro raíces trimestralmente, durante un año, en diez estaciones de muestreo. También se midió la temperatura del agua, salinidad, oxígeno disuelto, profundidad y transparencia en cada muestreo. Se determinó la abundancia, la biomasa y la riqueza específica, a lo largo de las raíces en segmentos verticales de $10 \mathrm{~cm}$ llamados horizontes. Se registraron un total de 59632 especímenes y una biomasa total de $172 \mathrm{~g} /$ $\mathrm{cm}^{2}$, y un máximo de once horizontes presentaron al menos una especie de epibionte. Se identificaron dos especies de poliquetos, 12 de moluscos y 14 de crustáceos, siendo Mytilopsis leucophaeata la más abundante, Ficopomatus miamiensis la más común y Crassostrea rhizophorae la de mayor contribución a la biomasa. Se evaluó la eficiencia del muestreo mediante curvas de acumulación de especies, resultando que la eficiencia fue, en general, $>90 \%$ de la riqueza estimada por modelos asintóticos. La hidrodinámica de la boca estuarina tuvo gran influencia en las condiciones ambientales de la laguna y en las variaciones espacio-temporales de la comunidad de epibiontes: (a) cuando la boca estuarina está cerrada son más claros los gradientes ambientales norte-sur, (b) cuando la boca estuarina se cierra y el nivel de inundación se incrementa, los epibiontes, especialmente la especie pionera $F$. miamiensis, ocupan los horizontes más altos de las raíces, (c) la abundancia, biomasa y riqueza específica de los epibiontes se incrementó hacia las partes más profundas del sur de la laguna, así como en los horizontes permanentemente inundados, y durante el cierre estacional de la boca estuarina. Las variaciones en la profundidad dependen de la dinámica de la boca estuarina, sugiriendo que estos factores son los principales parámetros que estructuran a la comunidad de epibiontes de La Mancha.

Palabras clave: boca estuarina efímera, profundidad, abundancia, biomasa, riqueza específica, crustáceos, moluscos, poliquetos, curvas de acumulación de especies.

\section{REFERENCIAS}

Abbott, R. T. (1974). American seashells. Second edition. USA: Van Nostrand Reinhold Co.

Acosta Balbas, V., Betancourt Tineo, R., \& Prieto Arcas, A. (2014). Estructura comunitaria de bivalvos y gasterópodos en raíces del mangle rojo Rhizophora mangle (Rhizophoraceae) en Isla Larga, bahía de Mochima, Venezuela. Revista de Biología Tropical, 62(2), 551-565.

Brusca, R. \& Brusca, G. J. (1990). Invertebrates. Massachussets, USA: Sinauer Associates, Inc. Sunderland.

Cedeño, J., Jiménez Prieto, M., Pereda, L., \& Allen, T. (2010). Abundancia y riqueza de moluscos y crustáceos asociados a las raíces sumergidas del mangle rojo (Rhizophora mangle) en la laguna de Bocaripo, Sucre, Venezuela. Revista de Biología Tropical, 58 (suppl. 3), 213-226.

Colwell, R. K. (1997). EstimateS: statistical estimation of species richness and shared species from samples. Version 5. USA: Department of Ecology and Evolutionary Biology, University of Connecticut. Recuperado de http://viceroy.eeb.uconn.edu/estimates.

Conde, J. E. \& Díaz, H. (1989). The mangrove tree crab Aratus pisonii in a tropical estuarine coastal lagoon. Estuarine, Coastal and Shelf Science, 28, 639-650.

Contreras Espinosa, F. (1993). Ecosistemas costeros mexicanos. México: CONABIO-UAM.

Creary, M. M. (2003). Spatial distribution of epibenthic bryozoans found on the roots of Rhizophora mangle, Kingston Harbour, Jamaica, WI. Bulletin of Marine Science, 73(2), 477-490. 
Cruz Soto, R. A. \& Jiménez, J. A. (1994). Moluscos asociados a las áreas de manglar de la Costa Pacífica de América Central: guía. Heredia, Costa Rica: Editorial Fundación UNA.

de la Lanza Espino, G. (1994). Química de las lagunas costeras y el litoral mexicano. In G. de la Lanza Espino \& C. Cáceres Martínez (Eds.), Lagunas Costeras y el litoral mexicano (pp. 127-198). México: Universidad Autónoma de Baja California Sur.

Díaz Díaz, O., Liñero Arana, I., Villafranca, S., \& Allen, T. (2009). Epizoic polychaetes (Annelida: Polychaeta) on Crassostrea rhizophorae (Guilding, 1828) from La Restinga Lagoon, Margarita Island, Venezuela. Ecotrópicos, 22(1), 13-22.

Díaz, M. C. \& Rützler, K. (2009). Biodiversity and abundance of sponges in Caribbean mangrove: indicators of environmental quality. Smithsonian Contributions of Marine Science, 38, 151-172.

Ellison, A. M. \& Farnsworth, E. J. (1992). The ecology of Belizean mangrove-root fouling communities: patterns of epibiont distribution and abundance, and effects on root growth. Hydrobiologia, 247, 87-98.

Ellison, A. M., Farnsworth, E. J., \& Twilley, R. R. (1996). Facultative mutualism between red mangroves and root-fouling sponges in Belizean mangal. Ecology, 77, 2431-2444.

Farnsworth, E. J. \& Ellison, A. M. (1996). Scale-dependent spatial and temporal variability in biogeography of mangrove root epibiont communities. Ecological Monographs, 66, 45-66.

Felder, D. L. (1973). An annotated key to crabs and lobsters (Decapoda: Reptantia) from coastal waters of the northwestern Gulf of Mexico. Louisiana, USA: Louisiana St. University, Sea Grant Publ.

Flores Andolais, F., García Cubas, A., \& Toledano Granados, A. (1988). Sistemática y algunos aspectos ecológicos de los moluscos de la laguna de La Mancha, Veracruz, México. Anales del Instituto de Ciencias del Mar y Limnología de la Universidad Nacional Autónoma de México, 15, 235-258.

García, E. (1988). Modificaciones al sistema de clasificación climática de Köppen. México: Instituto de Geografía, Universidad Nacional Autónoma de México.

García Cubas, A. (1981). Moluscos de un sistema lagunar tropical en el sur del Golfo de México (Laguna de Términos, Campeche). Anales del Instituto de Ciencias del Mar y Limnología de la Universidad Nacional Autónoma de México, 5, 1-182.

García Guerrero, M. \& Hendrickx, M. E. (2003). Distribution of isopods (Peracarida, Isopoda) associated with prop roots of Rhizophora mangle in a tropical coastal lagoon, southeastern Gulf of California, Mexico. Crustaceana, 76, 1153-1169.
García Padilla, J. M. \& Palacio, J. (2008). Macroinvertebrados asociados a las raíces sumergidas del mangle rojo (Rhizophora mangle) en las bahías Turbo y El Uno, Golfo de Urabá (Caribe Colombiano). Gestión y Ambiente, 11(3), 55-66.

Gaviño, G., Juárez, C., \& Figueroa, H. H. (1992). Técnicas biológicas selectas de laboratorio y de campo. Méxi$c o$ : Editorial LIMUSA.

Guerra Castro, E., Cruz Motta, J. J., \& Eloy, J. E. (2011). Cuantificación de la diversidad de especies incrustantes asociadas a las raíces de Rhizophora mangle L. en el Parque Nacional Laguna de la Restinga. Interciencia, 36(12), 923-930.

Hernández-Trejo, H., Priego Santander, Á. G., LópezPortillo, J., \& Insunza Vera, E. (2006). Los paisajes físico-geográficos de los manglares de la laguna de La Mancha, Veracruz, México. Interciencia, 31, 211-219.

Hogarth, P. J. (1999). The biology of mangroves. USA: Oxford University Press.

Laine, A. O., Mattila, J., \& Lehikoinen, A. (2006). First record of the brackish water dreissenid bivalve Mytilopsis leucophaeata in Northern Baltic Sea. Aquatic Invasions, 1(1), 38-41.

Lankford, R.R. (1977). Coastal lagoons of Mexico. Their origin and classification. In M. Wiley (Ed.), Estuarine Processes (pp. 245-254). New York, USA: Academic Press, Inc.

Lara Domínguez, A. L., Day, J. W., Yáñez Arancibia, A., \& Sáinz Hernández, E. (2006). A dynamic characterization of water flux through a tropical ephemeral inlet, La Mancha Lagoon, Gulf of Mexico. In V.P. Singh \& Y. Jun Xu (Eds.), Coastal Hydrology and Processes (pp. 413-422). USA: Water Resources Publications, LLC.

Little, C. \& Kitching. J. A. (1996). The biology of rocky shores. USA: Oxford University Press.

Londoño Mesa, M., Polanía, J., \& Vélez, I. (2002). Polychaetes of the mangrove-fouling community at the Colombian Archipelago of San Andrés and Old Providence, Western Caribbean. Wetlands Ecology and Management, 10, 227-232.

Marelli, D. C. \& Gray, S. (1983). Conchological redescriptions of Mytilopsis sallei and Mytilopsis leucophaeata of the brackish Western Atlantic (Bivalvia: Dreissenidae). Veliger, 25, 185-193.

Márquez, B. \& Jiménez, M. (2002). Moluscos asociados a las raíces sumergidas del mangle rojo Rhizophora mangle, en el Golfo de Santa Fe, Estado de Sucre, Venezuela. Revista de Biología Tropical, 50(3/4), 1101-1112.

Moreno, C. E. \& Halffter, G. (2000). Assessing the completeness of bat biodiversity inventories using species 
accumulation curves. Journal of Applied Ecology, 37, 149-158.

Ortiz, L. F. \& Blanco, J. F. (2012). Distribución de los gasterópodos del manglar, Neritina virginea (Neritidae) y Littoraria angulifera (Littorinidae) en la Ecorregión Darién, Caribe colombiano. Revista de Biología Tropical, 60(1), 219-232.

Prüsmann, J. \& Palacio, J. (2008). Colonización de moluscos y crustáceos en las raíces de mangle rojo en una laguna costera de la punta norte del golfo de Morrosquillo. Gestión y Ambiente, 11(3), 77-86.

Quiceno Cuartas, P. A. \& Palacio Baena, J. A. (2008). Aporte al conocimiento de los macroinvertebrados asociados a las raíces del mangle (Rhizophora mangle) en la Ciénaga La Boquilla, municipio de San Onofre, Sucre. Gestión y Ambiente, 11(3), 67-78.

Quirós, J. A. \& Arias, J. E. (2013). Taxocenosis de moluscos y crustáceos en raíces de Rhizophora mangle (Rhizophoraceae) en la bahía de Cispatá, Córdoba, Colombia. Acta biológica Colombiana, 18(2), 329-340. Recuperado de http://www.redalyc. org/pdf/3190/319028011009.pdf.

Rioja, E. (1945). Estudios anelidológicos. XII. Un nuevo género de serpúlido de agua salobre de México. Anales del Instituto de Biología de la Universidad Nacional Autónoma de México, 16, 411-417.

Rioja, E. (1946). Estudios anelidológicos. XV. Nereidos de agua salobre de los esteros del litoral del Golfo de México. Anales del Instituto de Biología de la Universidad Nacional Autónoma de México, 17, 205-214.

Romero Murillo, P. E. \& Polanía, J. (2008). Sucesión temprana de la taxocenosis Mollusca-Annelida-Crustacea en raíces sumergidas de mangle rojo en San Andrés Isla, Caribe Colombiano. Revista de Biología Marina y Oceanografia, 43(1), 63-74.

Rützler, K., \& Feller, C. (1996). Caribbean mangrove swamps. Scientific American, 274, 70-75.

Rützler, K. \& Feller, C. (1999). Mangrove swamp communities: an approach in Belize. In A. Yáñez-Arancibia \& A. L. Lara-Domínguez (Eds.), Ecosistemas de manglar en América Tropical (pp. 39-50). Xalapa, México: Instituto de Ecología, A.C., UICN/ORMA, y NOAA/NMFS Silver Spring MD.

SAGARPA (2013, 18 de diciembre). Acuerdo por el que se dan a conocer las Reglas de Operación del Programa de Fomento a la Productividad Pesquera y Acuícola de la Secretaría de Agricultura, Ganadería, Desarrollo Rural, Pesca y Alimentación. Diario Oficial de la Federación. Recuperado de http://www.sagarpa.gob. mx/-programassagarpa/documents/rop2014-ves.pdf

Salgado Barragán, J. \& Hendrickx, M. E. (2002a). Distribution and abundance of barnacles (Crustacea:
Cirripedia) associated with prop roots of Rhizophora mangle L. in a lagoon of Northwest Mexico. In M. E. Hendrickx (Ed.), Contributions to the study of East Pacific Crustaceans (pp. 45-60). México: Instituto de Ciencias del Mar y Limnología, UNAM.

Salgado Barragán, J. \& Hendrickx, M. E. (2002b). Panopeid crabs (Crustacea: Brachyura: Panopeidae) associated with prop roots of Rhizophora mangle L. in a tropical coastal lagoon of the SE Gulf of California, México. In E. Escobar-Briones \& F. Alvarez (Eds.), Modern approaches to the study of Crustacea (pp. 1-7). USA: Kluwer Academic/Plenum Publishers.

Schultz, G. A. (1969). How to know the marine isopod crustaceans. Dubuque, Iowa, USA: WM. C. Brown Co. Publishers.

Soberón, J. M. \& Llorente, J. B. (1993). The use of species accumulation functions for the prediction of species richness. Conservation Biology, 7, 480-488.

Sokal, R. \& Rohlf, F. (1979). Biometría y principios y métodos estadísticos en la investigación biológica. Madrid, España: Blum.

Souza, J. R. B. de, Rocha, C. M. C. da, \& Lima, M. dos P. R. de. (2005). Ocorrencia do bivalve exótico Mytilopsis leucophaeata (Conrad) (Mollusca, Bivalvia), no Brasil. Revista Brasileira de Zoología, 22(4), 1204-1206.

Sutherland, J. P. (1980). Dynamics of the epibenthic community on roots of the mangrove Rhizophora mangle, at Bahia de Buche, Venezuela. Marine Biology, 58, 75-84.

Ter Braak, C. J. F. (1986). Canonical Correspondence Analysis: A new eigenvector technique for multivariate direct gradient analysis. Ecology, 67(5), 1167-1179.

Twilley, R. R., Snedaker, S. C., Yáñez Arancibia, A., \& Medina, E. (1996). Biodiversity and ecosystem processes in tropical estuaries: perspectives of mangrove ecosystems. In H. A. Mooney, J. H. Cushman, E. Medina, O. E. Sala, \& E. D. Schulze (Eds.), Functional roles of biodiversity: a global perspective (pp. 328-370). USA: ICSU, UNEP, John Wiley \& Sons Ltd.

Vilardy, S. \& Polanía, J. (2002). Mollusc fauna of the mangrove root fouling community at the Colombian Archipelago of San Andrés and Old Providence. Wetlands Ecology and Management, 10, 273-282.

Villalobos Figueroa, A., Zamora, M. E., De la Parra, R., Cacho, O., Galván, V., \& Izaguirre, M. A. (1984). Estudio hidrobiológico en la laguna de La Mancha, Mpio. de Actopan, Ver. 1979-1980. Boletín del Instituto Nacional de Investigaciones sobre Recursos Bióticos, 15, 1-51. 
Williams, A. B. (1983). The mud crab, Panopeus herbstii S. L. partition into six species (Decapoda: Xanthidae). Fishery Bulletin, 81, 863-882.

Williams, A. B. (1984). Shrimps, lobsters, and crabs of the Atlantic coast of the eastern United States, Maine to Florida. Washington, DC: Smithsonian Institution Press.

Wilson, K. A. (1981). Tidal-associated feeding in the mangrove tree crab, Aratus pisonii. American Zoologist, 21,1005 .
Wulff, J. L. (2012). Ecological interactions and the distribution, abundance and diversity of sponges. Advances in Marine Biology, 61, 273-344.

Yáñez Arancibia, A. \& Day, J. W. (2005). Ecosystem Functioning: The basis for sustainable management of Terminos Lagoon, Campeche, Mexico. Xalapa, Veracruz, Mexico: Instituto de Ecología, A.C.

Zar, J. H. (1996). Biostatistical analysis. Third edition. New Jersey, USA: Prentice Hall. 